\title{
Optimal Electric Bus Scheduling under Travel Time Uncertainty: A Robust Model and Solution Method
}

\author{
Mengyan Jiang $\mathbb{D}^{1}{ }^{1}$ Yi Zhang $\mathbb{D}^{1,2}$ and Yi Zhang $\mathbb{D}^{1,3}$ \\ ${ }^{1}$ Center of Environmental Science and New Energy Technology, Tsinghua-Berkeley Shenzhen Institute, Tsinghua University, \\ Shenzhen 518055, China \\ ${ }^{2}$ Department of Automation, Tsinghua National Laboratory for Information Science and Technology (TNList), \\ Tsinghua University, Beijing 100084, China \\ ${ }^{3}$ Institute of Future Human Habitats, Shenzhen International Graduate School, Tsinghua University, Shenzhen 518055, China
}

Correspondence should be addressed to Yi Zhang; zy1214@sz.tsinghua.edu.cn

Received 22 October 2021; Revised 18 November 2021; Accepted 24 November 2021; Published 20 December 2021

Academic Editor: Wenxiang Li

Copyright $(02021$ Mengyan Jiang et al. This is an open access article distributed under the Creative Commons Attribution License, which permits unrestricted use, distribution, and reproduction in any medium, provided the original work is properly cited.

With the increasing adoption of electric buses (e-buses), e-bus scheduling problem has become an essential part of transit operation planning. As e-buses have a limited battery capacity, e-bus scheduling problem aims to assign vehicles to timetabled service trips on the bus routes considering their charging demand. Affected by the dynamic operation environment, the travel time and energy consumption of the e-buses often display considerable randomness, resulting in unexpected trip start delays and battery energy shortages. In this paper, we addressed the e-bus scheduling problem under travel time uncertainty by robust optimization approaches. We consider the cardinality constrained uncertainty set to formulate a robust multidepot EVSP model considering trip time uncertainty and partial recharging. The model is developed based on the dynamic programming equations that we formulated for trip chain robustness checking. A branch-and-price (BP) algorithm is devised to generate provably highquality solutions for large-scale instances. In the BP algorithm, an efficient label setting algorithm is developed to solve the robust resource-constrained shortest path subproblem. Comprehensive numerical experiments are conducted based on the bus routes in Shenzhen to demonstrate the effectiveness of the suggested methodology. The robustness of the schedules was evaluated through Monte Carlo simulation. The results show that the trip start delay and battery energy shortage caused by the travel time uncertainty can be effectively reduced at the expense of an increase in the operational cost. A trade-off should be made between the reduction in infeasibility rate and increase in operational cost to choose a proper uncertainty budget.

\section{Introduction}

In recent years, an increasing number of electric buses (e-buses) have been introduced into the public transit systems because of their environmental and social benefits such as reducing on-road pollution, energy saving, and better onboard experience [1]. However, as the driving range of the e-buses per charge is limited and recharging the battery is time-consuming, the vehicles need extra time for battery recharging during the operation. To ensure operational efficiency, a charging plan for the e-bus fleet is required which specifies the optimal time, location, and amount to charge. In the operation planning phase, the electric vehicle scheduling problem (EVSP) aims to assign timetabled trips to the e-buses while making optimal charging plans to minimize the operational cost.

Two essential parameters taken as input to the EVSP are the expected travel time and energy consumption of the bus trips which have a great impact on the reliability of the schedule. In real-life cases, getting accurate estimations of the trip travel time is difficult because of the substantial variability of traffic condition, passenger demand, and driving conditions. Consequently, the energy consumption of a trip can also deviate from the estimated value considerably. To mitigate possible variations, on the operation planning side, the operators usually introduce a buffer time between consecutive trips to absorb small delays and a safe range for the battery state of charge (SoC). However, buffer 
time is usually very short and not able to protect the schedule against trip time variations effectively. If some trips experience longer travel time than the expected value, the delay will propagate along the trip chain and influence the punctuality of the consecutive trips. The original charging plan can also become infeasible as the charging time and vehicle battery SoC are also influenced by the trip travel time.

With the aim to generate robust schedules against trip travel time variation and reduce the delays that cannot be absorbed by the buffer time, the stochastic vehicle scheduling model and dynamic rescheduling strategies are proposed in the literature [2-7]. Different from the exiting studies, we aim to tackle the travel time uncertainty in the EVSP by robust optimization methods. The optimized schedule can remain feasible regarding the trip start time and vehicle battery SoC when trip time varies in the budgeted uncertainty set. The main contribution of this study is as follows:

(i) We consider the cardinality constrained uncertainty set to formulate a robust multidepot EVSP (R-MDEVSP) model considering trip time uncertainty and partial recharging. The R-MD-EVSP model is developed based on the dynamic programming (DP) equations that we formulated for individual trip chain robustness checking.

(ii) A branch-and-price (BP) algorithm is devised where the pricing subproblem, a robust resource-constrained shortest path problem, is solved by an efficient labeling algorithm based on the DP equations.

(iii) Comprehensive numerical experiments are conducted based on the cases in Shenzhen with hundreds of trips on multiple bus routes. The robustness of the robust solutions is verified through Monte Carlo simulations.

The remainder of the paper is organized as follows. In the next section, a literature review on the EVSP is provided. Section 3 proposes the DP equations for trip chain robustness checking and the robust EVSP formulation. The BP algorithm is described in Section 4. The results of the numerical experiments are presented in Section 5. We conclude the paper in Section 6.

\section{Literature Review}

In the operation planning stage of public transportation, the vehicle scheduling problem (VSP) aims at allocating transit vehicles to carry out timetabled trips with the objective to minimize the total number of vehicles used. Single-depot VSP is polynomial time solvable while multidepot VSP has proven to be NP-hard by Bertossi et al. [8]. With the wide adoption of e-buses in the transit system in recent years, EVSP arises under different scenarios regarding the charging technology, charging policy, and fleet composition. Li [9] and Yang et al. [10] developed models and algorithms for the EVSP under battery swapping modes. Considering the plug- in charging mode, Liu and Ceder [11] proposed a model based on the deficit function theory aimed at minimizing the number of vehicles and chargers. Mixed fleet EVSP was considered in [12-14]. Zhang et al. [15] addressed an EVSP considering the degradation of battery and nonlinear charging process. A tailored BP algorithm was devised to solve the problem. Their computational experiments showed that the optimized schedule can reduce the cost considerably which is mainly achieved by the substantial extension of battery life. Considering nonlinear charging process and multivehicle type, Zhang et al. [16] developed an MIP model for the EVSP with linear approximation of the nonlinear charging function. An ALNS heuristic was devised to solve the problem.

The above studies assume a full charging policy, while allowing for partial charging increases the operational flexibility and also the problem complexity. Considering partial charging, Wen et al. [17] developed a multidepot EVSP and an adaptive large neighborhood search heuristic to solve instances with customized bus trips. van Kooten Niekerk et al. [18] proposed two models for single-depot scenarios with a different level of detail resembling the actual charging processes. A column generation algorithm was developed to solve the problem. Koháni and Kohánia [19] proposed a linear MIP model for a single-depot EVSP considering the location of the charging stations and the assignment of chargers to the vehicles. The model was solved by the standard solver. Li et al. [20] addressed EVSP along with the charger deployment problem. An adaptive genetic algorithm is designed to solve the problem. Yildirım and Y1ldiz [21] considered an optimal fleet composition and scheduling problem. An IP-column-generation algorithm is proposed to solve the pricing subproblem.

Charging scheduling problem aims to determine the time, amount, and specific charger for each e-bus to get recharged. In large-sized transit systems, due to the problem scale and complexity, charging scheduling often assumes that the e-bus operation schedule is given beforehand. Qin et al. [22] conducted a simulation analysis on the daily charging patterns and demand charges of a fleet of e-buses. An optimal charging strategy is identified to minimize demand charges. Wang et al. [23] developed a mixed-integer programming (MIP) model for the e-bus recharging scheduling based on a real-world case. The model aims to assign the charging station, chargers, and charging time to the e-buses to minimize the system operating costs, assuming given timetable and operation schedule. With the goal to minimize the electricity demand charges and energy charges, $\mathrm{He}$ et al. [24] proposed a modeling framework to determine the time to charge a vehicle and the actual charging power. Liu et al. [25] considered the e-bus charging scheduling problem under limited charging resources in the charging station. A column-generation-based algorithm is developed to solve the large-scale problem instances. The results show that the optimal charging plan can greatly reduce the charging cost compared with the uncontrolled charging.

Most of the studies take deterministic trip time as the model input and assume that the energy discharge is 
proportional to the travel distance. To tackle the trip time uncertainty in the real world, on the input side, some methods were proposed to improve the accuracy of the trip time estimation based on historical running data [26, 27]. On the planning side, dynamic rescheduling strategies and stochastic VSP models were developed. Huisman et al. [3] introduced a dynamic vehicle scheduling approach to solve the VSP periodically with renewed estimation on the future trip time. He et al. [5] formulated a stochastic dynamic VSP and adopted an approximate dynamic programming approach where the objective function is approximated by a feed-forward neural network. Huisman et al. [3] developed a stochastic VSP model using typical disruption scenarios to minimize the expected total planned costs and costs caused by disruptions. Shen et al. [2] proposed a probabilistic network flow model assuming certain trip time distributions and developed a hybrid heuristic solution method. Considering the stochastic travel time, Tang et al. [6] proposed a stochastic model and a dynamic rescheduling paradigm for a single-depot EVSP with the full charging policy. A speedenergy-consumption relationship was incorporated in their models. Bie et al. [7] proposed a multiobjective stochastic e-bus scheduling model considering the variability of travel time and energy consumption. The numerical study showed that the optimized schedule can effectively protect against the accumulation of stochastic volatilities.

Compared with the EVSP, more studies have considered the travel time uncertainty in the vehicle routing problem (VRP). VRP arises in the logistics context which aims to plan for vehicle routes to serve customers at different locations. To tackle the uncertainty related to customer demand and vehicle travel time, stochastic and robust models as well as dynamic reoptimization approaches were proposed in the literature. The advantage of robust optimization is that it only requires defined bounds on the data rather than the underlying distribution while maintaining the tractability of the problem. Different kinds of uncertainty sets have been considered in robust VRP (RVRP) with the typical ones including the hypercube, budgeted uncertainty sets, and ellipsoidal sets. The cardinality constrained set proposed by Bertsimas and Sim [28] is widely used which specifies an upper bound of the number of customer demand or the travel links that can attain their maximum value.

Motivated by the travel time uncertainty in maritime transportation, Agra et al. [29] proposed two RVSP formulations. Dynamic programming recursive equations were proposed for path feasibility checking based on which path inequalities can be generated. They showed that it suffices to consider a subset of the extreme points of the uncertainty polytope and developed scenario reduction solution techniques. Salicru et al. [26] proposed a compact formulation for the RVRP under demand uncertainty with dynamic programming equations integrated. To solve the RVRP and its variants, exact algorithms were developed based on the branchprice-and-cut method by reformulating the robust problem into its deterministic counterpart [30, 31]. Local search-based heuristic frameworks with solution robustness checking procedure embedded in were devised in $[32,33]$ to solve largesized instances. Pelletier et al. [34] considered a capacitated electric VRP with energy consumption uncertainty. They considered different kinds of uncertainty sets and proposed an LNS-based heuristic to solve the problem.

From the above discussion, we can see that although many studies have focused on the EVSP, few of them have considered the uncertainty related to the travel time and energy consumption in the problem. To the best of our knowledge, there has been no research to cope with the trip time uncertainty of the EVSP using the robust optimization method. Our study proposed a robust EVSP model and solution method considering the travel time uncertainty under budgeted uncertainty set.

\section{Robust Multidepot EVSP}

3.1. Problem Description. A bus route in the transit network is defined by two end stops and a series of intermediate stops. On a bus route, a service trip starts from one end stop at a scheduled time and ends at the other end stop to carry passengers. The timetable of the route includes all the trips that should be carried out in one day with their scheduled start time from the start stop and expected end time at the end stop. On the electrified bus routes, these trips are carried out by a fleet of e-buses. Each e-bus undertakes a sequence of trips in a day which is called a trip chain. An operation schedule consists of the trip chains for all the e-buses. For the convenience of operation, bus depots are established close to the end stops of the routes for vehicle parking, maintenance, and charging if charging facilities are established. Based on this setting, the R-MD-EVSP is described as follows. The list of notations is provided in Table 1 .

Let $G=(V, A)$ be an acyclic direct graph, where $V$ is the set of nodes and $A$ is the set of arcs. Each timetabled trip is represented by a trip node in the graph. Denote $T \subseteq V$ as the set of trip nodes. Each trip node $i \in T$ is associated with a trip start time window $\left[a_{i}, b_{i}\right]$, where $a_{i}$ and $b_{i}$ are the scheduled start time and latest start time, respectively. The e-bus serving a trip should wait until the scheduled start time $a_{i}$ if it arrives earlier at the trip start depot. Each trip node $i \in T$ is associated with two uncertain parameters: the trip time $\tau_{i}$ and trip energy consumption $\varepsilon_{i} . \tau_{i}$ is realized in the interval $\left[t_{i}-\widetilde{t}_{i}, t_{i}+\widehat{t}_{i}\right]$ where $t_{i}$ is the nominal trip time; $\widehat{t}_{i} \geq 0$ and $\tilde{t}_{i} \geq 0$ are the upper and lower deviations from $t_{i} . \varepsilon_{i}$ is realized in the interval $\left[e_{i}-\widetilde{e}_{i}, e_{i}+\widehat{e}_{i}\right]$ where $e_{i}$ is the nominal value; $\widehat{e}_{i} \geq 0$ and $\widetilde{e}_{i} \geq 0$ are the upper and lower deviations from $e_{i}$. Since the worst case will always be achieved at the right-hand side of the interval, we only consider the realization of $\tau_{i}$ in $\left[t_{i}, t_{i}+\widehat{t}_{i}\right]$ and $\varepsilon_{i}$ in $\left[e_{i}, e_{i}+\widehat{e}_{i}\right]$ correspondingly.

Denote $K$ as the set of depots. Each depot $k \in K$ is created with two nodes in the graph: operation start node $o^{k}$ and operation end node $d^{k}$, indicating that the e-bus begins/ ends the operation from/at depot $k$, respectively. For nodes $o^{k}$ and $d^{k}, k \in K$, we assign a time window $\left[a_{0}, b_{0}\right]$, where $a_{o}$ is the earliest operation start time and $b_{0}$ is the latest operation end time. This means that the e-buses can begin and end their operation at any time within time window $\left[a_{0}, b_{0}\right]$.

The arc set $A$ includes three kinds of arcs: (i) the pull-out arc connects a depot node $o^{k}, k \in K$, and a trip node $i \in T$, representing that a vehicle begins operation from depot $k$ to 
TABLe 1: Definitions of the sets and parameters for the R-MD-EVSP model.

\begin{tabular}{|c|c|}
\hline Sets & \\
\hline K & Set of depots with index $k$ \\
\hline$V_{t}$ & Set of trip nodes \\
\hline$V^{t}$ & Set of nodes \\
\hline$A$ & Set of arcs \\
\hline Paran & \\
\hline$a_{0}$ & Earliest operation start time \\
\hline$b_{0}$ & Latest operation end time \\
\hline$a_{i}$ & Scheduled departure time of trip node $i$ \\
\hline$b_{i}$ & Latest departure time of trip node $i$ \\
\hline$\tau_{i}$ & Trip time of trip node $i$, an uncertain parameter \\
\hline$t_{i}$ & Nominal trip time of trip node $i$ \\
\hline$\widehat{t}_{i}$ & Upper trip time deviation from $t_{i}$ \\
\hline$\tilde{t}_{i}$ & Lower trip time deviation from $t_{i}$ \\
\hline$\delta_{i}^{t}$ & Trip time deviation of trip node $i, \delta_{i}^{t} \in\left[0, \widehat{t}_{i}\right]$ \\
\hline$\varepsilon_{i}$ & Trip energy consumption of trip node $i$, an uncertain parameter \\
\hline$e_{i}$ & Nominal energy consumption of trip node $i$ \\
\hline$\widehat{e}_{i}$ & Upper energy consumption deviation from $e_{i}$ \\
\hline$\tilde{e}_{i}$ & Lower energy consumption deviation from $e_{i}$ \\
\hline$\delta_{i}^{e}$ & Energy consumption deviation of trip node $i, \delta_{i}^{e} \in\left[0, \widehat{e}_{i}\right]$ \\
\hline$s_{i j}$ & Empty travel time from node $i$ to node $j$ \\
\hline$e_{i j}$ & Energy consumption of the empty travel from node $i$ to node $j$ \\
\hline$c_{i j}$ & Cost of $\operatorname{arc}(i, j) \in A$ \\
\hline$c^{i j}$ & Departure delay cost per minute \\
\hline$c^{e}$ & Unit charging cost \\
\hline$t^{p}$ & Charging preparation time \\
\hline$r_{s}$ & Energy charging rate \\
\hline$r_{u}$ & Energy consumption rate \\
\hline$\Gamma^{t}$ & Uncertainty budget, maximum number of trips that can take their upper bound trip time, indexed by $\gamma$ \\
\hline$u^{\max }$ & Upper bound of the vehicle battery SoC \\
\hline$u^{\min }$ & Lower bound of the vehicle battery SoC \\
\hline$u_{i \gamma}^{\min }$ & Lower bound of variable $Y_{i \gamma}, \gamma \leq \Gamma^{t}$ \\
\hline
\end{tabular}

carry out the first trip $i$; (ii) the pull-in arc connects a trip node $i \in T$ and an end node $d^{k}, k \in K$, representing that a vehicle finishes its last trip $i$ and ends the operation, returning to depot $k$; and (iii) the trip connection arc connects two trip nodes, representing that two trips are carried out consecutively. Each arc $(i, j) \in A$ is associated with a nonservice travel time $s_{i j}$ and energy consumption $e_{i j}$. To ensure that the trip start time window is respected, trip nodes $i$ and $j, i, j \in T$, are connected only if the time compatible condition $a_{i}+t_{i}+s_{i j} \leq b_{j}$ is satisfied. The cost of an $\operatorname{arc} c_{i j}$ includes the empty travel cost and the vehicle usage cost $f$ for the pull-out arcs. Denote $g_{i j},(i, j) \in A$, as the unit charging cost in the time interval between the end of trip node $i$ and the start of trip node $j ; g_{i j}$ is calculated as the weighted average value of the time-of-use tariff in the time interval.

Figure 1 gives an example of the graph for an instance with two depots and four trips. Each depot is created with a start node $o^{k}$ and an end node $d^{k}, k=1,2$. Each trip is represented by a trip node $v_{i}$ associated with a departure time window and a scheduled trip time, $i=1,2,3,4$.

We assume that the e-buses begin the operation with a fully charged battery and get recharged at the destination depot after finishing a trip if needed. The charging amount is proportional to the charging time with a rate of $r_{s}(\mathrm{kWh} /$ min). Denote $t^{s}$ as the unit charging time and $t^{p}$ as the

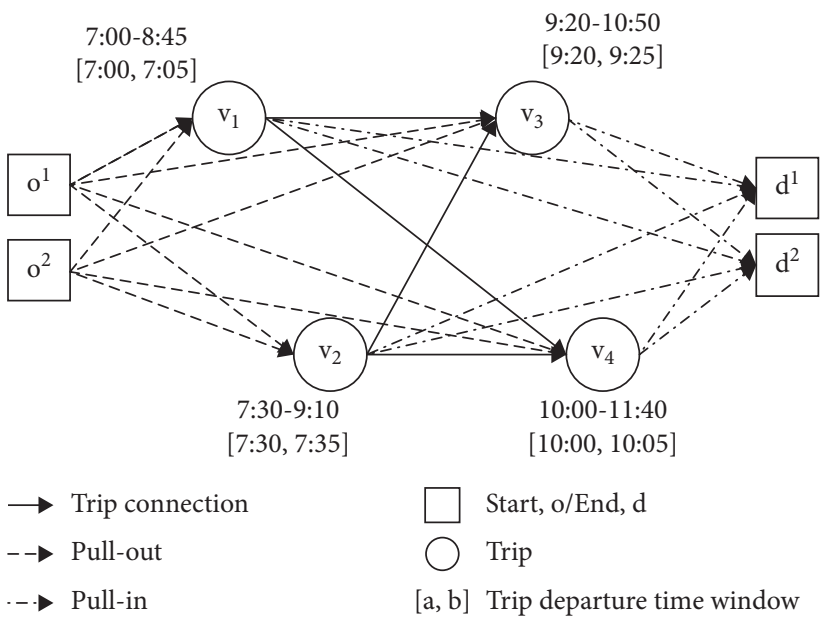

FIgURE 1: An illustrative example of the graph for the robust multidepot EVSP.

charging preparation time. The e-buses should charge integer times of $t^{s}$.

The historical running data of 150 bus routes in five months in Shenzhen were used to analyze the relationship between the trip time and trip energy consumption. Most of them display similar relationship. Figure 2 shows the 


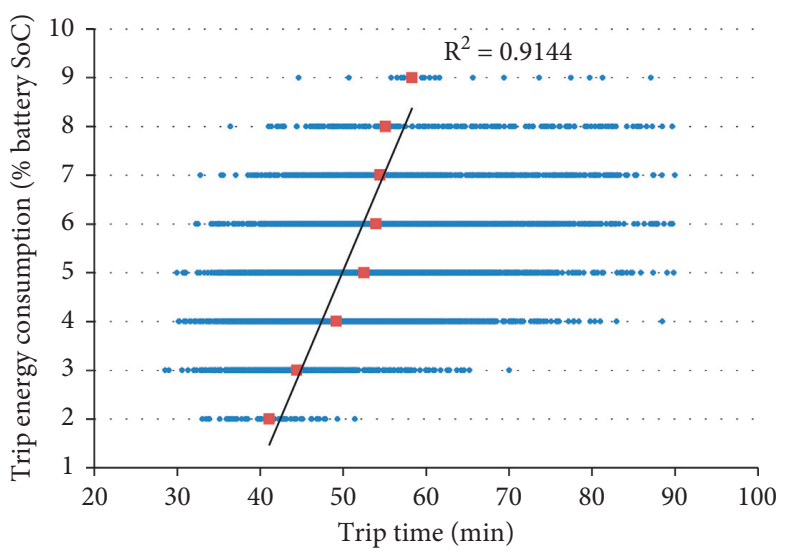

- Trip energy consumption sample

- Sample average

(a)

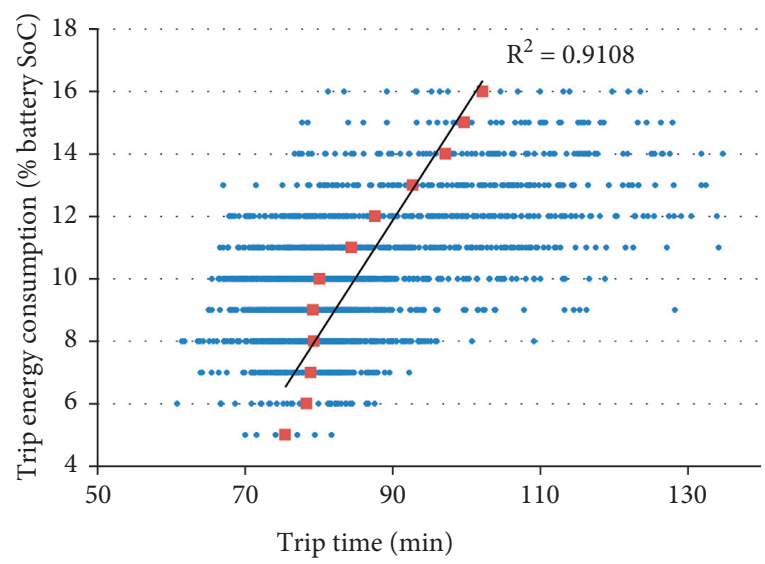

- Trip energy consumption sample

- Sample average

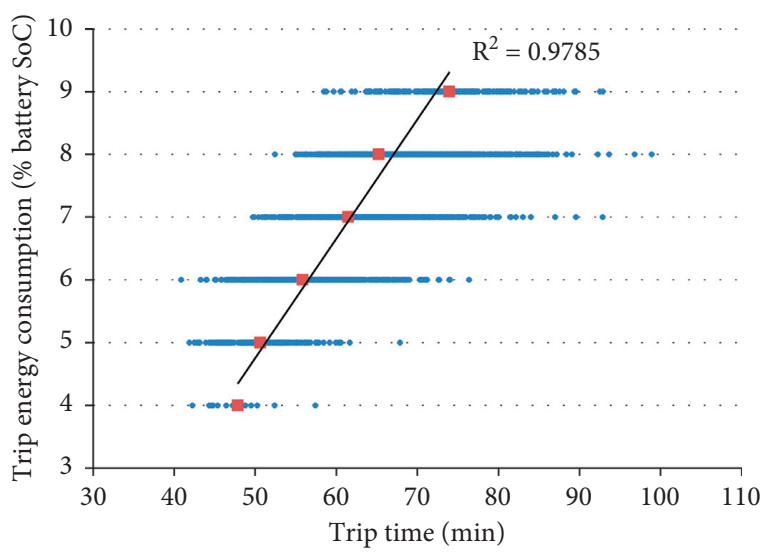

- Trip energy consumption sample

- Sample average

(b)

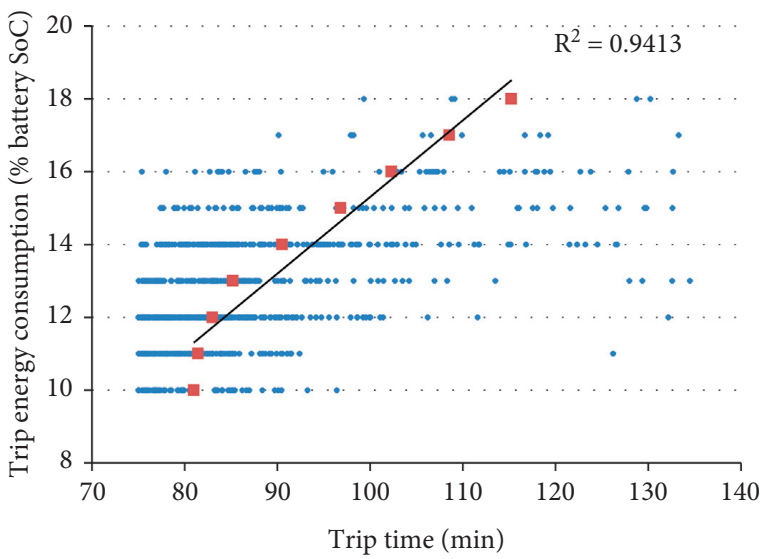

- Trip energy consumption sample

- Sample average

(d)

FIgURE 2: Relationship between the trip time and energy consumption for the selected bus routes. (a) Route 1. (b) Route 2. (c) Route 3. (d) Route 4.

relationship between the average trip time and energy consumption of the selected four bus routes. The solid lines are the linear regression results of the sample average data with the R-square values given next to it. The R-square values are both above 0.9 , meaning that the trip energy consumption can be described as a linear function of the trip time, although the data of Route 3 and Route 4 display some nonlinear trends. The trip time and trip energy consumption may display different relationships in different cities and routes. Based on the cases of Shenzhen, we assume that for a trip $i$, the energy consumption deviation $\delta_{i}^{e} \in\left[0, \widehat{e}_{i}\right]$ is proportional to the trip time deviation $\delta_{i}^{t} \in\left[0, \widehat{t}_{i}\right]$, i.e., $\delta_{i}^{e}=$ $\alpha \delta_{i}^{t} r_{u}$ where $\alpha \geq 1$ is an augment constant and $r_{u}$ is the energy consumption rate $(\mathrm{kWh} / \mathrm{min})$. Note that the robust EVSP model developed in this study does not depend on the assumption of a linear relationship between trip time and trip energy consumption. We assume that the energy consumption of a trip $i$ varies within interval $\left[e_{i}, e_{i}+\widehat{e}_{i}\right]$; as such, we only need to give the values of $e_{i}$ and $\hat{e}_{i}$ as the model inputs. Therefore, any empirical method that can give estimation on the values of $e_{i}$ and $\widehat{e}_{i}$ works.

We adopt a cardinality constrained uncertainty set $U$ as discussed in [24] to control the level of trip time uncertainty. $U$ is defined as $U=\left\{\tau \in \mathbb{R}^{|T|} \mid \tau_{i}=t_{i}+\theta_{i} \widehat{t}_{i}, 0 \leq \theta_{i} \leq 1, \forall i \in T\right.$, $\left.\sum_{i \in T} \theta_{i} \leq \Gamma^{t}\right\}$ where the cumulative uncertainty of the random variable $\theta_{i}$ is bounded by the budget $\Gamma^{t}$. We choose this uncertainty set because it is commonly used in the RVRP, the solution structure of which is similar to our problem: the trip chain assigned to an e-bus is equivalent to the customer route assigned to a vehicle in the VRP, where a bus trip can be regarded as a customer.

Based on the above settings, the problem looks for the minimum cost schedule that satisfies the following constraints: (i) each trip is carried out exactly once; (ii) the departure time window of each trip is respected when the trip time varies within the budgeted set $U$; (iii) each vehicle begins and ends its operation at the same depot; and (iv) the vehicle battery SoC is always kept within the safety range $\left[u^{\min }, u^{\max }\right]$ when the trip 
time varies within the budgeted set $U$. Following the robust optimization paradigm, a trip chain is robust feasible if it satisfies conditions (ii)-(iv) for all possible trip time realizations. A feasible schedule of the problem is given as set of feasible trip chains that together also satisfies condition (i).

3.2. Deterministic Problem Formulation. Let $X_{i j k}$ be the binary variables that take value 1 if a vehicle housed at terminal $k$ traverses arc $(i, j) \in A$. At each trip node $i \in T$, denote $Z_{i}$ as the trip start time and $Y_{i}$ as the lowest battery SoC of the vehicle at the trip start time. The lower bound of $Y_{i}$ is denoted as $u_{i}^{\text {min }}=u^{\text {min }}+e_{i}$. At each operation start (end) node $o^{k}\left(d^{k}\right)$, $k \in K$, denote $Z_{i}$ as the operation start (end) time and $Y_{i}$ as the lowest battery SoC of the vehicle at the operation start (end) time. On each arc $(i, j) \in A, i, j \in T$, denote $W_{i j}$ as the amount of energy to be charged after completing trip $i$ before starting trip $j$. According to our assumption, the charging place is at the ending depot of trip node $i . R_{i j}$ are the auxiliary variables defined corresponding to $Z_{i}$ for constraint linearization. Denote $E_{i j}$ as the binary variables that are equal to 1 if the e-buses can be charged on $\operatorname{arc}(i, j) \in A$. Denote $U_{i j}$ as the integer variables that specify the number of time units an e-bus spends charging on arc $(i, j) \in A$. The deterministic problem is formulated as follows (MD-EVSP):

$$
\begin{aligned}
& \min \sum_{k \in K} \sum_{(i, j) \in A} c_{i j} X_{i j k}+\sum_{(i, j) \in A} g_{i j} W_{i j} \\
& \text { s.t. } \sum_{k \in K} \sum_{j:(i, j) \in A} X_{i j k}=1, \quad i \in T \text {, } \\
& \sum_{j:(i, j) \in A} X_{i j k}-\sum_{j:(j, i) \in A} X_{j i k}=0, \quad i \in T, k \in K, \\
& \sum_{\beta \in K \backslash\{k\}} \sum_{i \in V} X_{o^{\beta} i k}=0, \quad k \in K, \\
& \sum_{\beta \in K \backslash\{k\}} \sum_{i \in V} X_{i d^{\beta} k}=0, \quad k \in K \\
& a_{i} \leq Z_{i} \leq b_{i}, \quad i \in V, \\
& Z_{j}-Z_{i}-t_{i}-s_{i j}+M\left(1-\sum_{k \in K} X_{i j k}\right) \geq 0,(i, j) \in A, \\
& Y_{i}-e_{i}+W_{i j}-e_{i j}+M\left(1-\sum_{k \in K} X_{i j k}\right) \geq Y_{j}, \quad(i, j) \in A, \\
& E_{i j}-\sum_{k \in K} X_{i j k} \leq 0,(i, j) \in A, \\
& -\left(a_{j}-Z_{i}-t_{i}-s_{i j}-t^{p}\right)-M\left(1-E_{i j}\right) \leq 0,(i, j) \in A,
\end{aligned}
$$

$$
\begin{aligned}
& W_{i j}+R_{i j} r_{s}-\left(a_{j}-t_{i}-s_{i j}-t^{p}\right) r_{s} E_{i j} \leq 0,(i, j) \in A, \\
& W_{i j}+Y_{i}-e_{i}-u^{\max } \leq 0,(i, j) \in A, \\
& W_{i j}-r_{s} t^{s} U_{i j}=0,(i, j) \in A, \\
& R_{i j}-b_{i} E_{i j} \leq 0,(i, j) \in A, \\
& a_{i} E_{i j}-R_{i j} \leq 0,(i, j) \in A, \\
& R_{i j}-Z_{i}+a_{i}\left(1-E_{i j}\right) \leq 0,(i, j) \in A, \\
& Z_{i}-b_{i}\left(1-E_{i j}\right)-R_{i j} \leq 0,(i, j) \in A, \\
& u_{i}^{\min } \leq Y_{i} \leq u^{\max }, \quad i \in V \\
& W_{i j} \geq 0,(i, j) \in A \text {, } \\
& X_{i j k} \in\{0,1\}, \quad(i, j) \in A, k \in K, \\
& E_{i j} \in\{0,1\}, \quad(i, j) \in A, \\
& R_{i j} \geq 0, \quad(i, j) \in A, \\
& U_{i j} \in Z, \quad(i, j) \in A .
\end{aligned}
$$

Objective (1) minimizes the sum of vehicle usage, empty travel, and charging cost. Constraint (2) ensures that each trip is carried out only once. Constraint (3) is the flow conservation constraint. Constraints (4) and (5) require that each vehicle begin and end its operation at its base depot. Constraint (6) limits the departure time of each trip to be within the departure time window. Constraint (7) ensures the time consistency of two successive trips. Constraint (8) ensures the vehicle battery energy consistency of two successive trips. Constraint (9) requires that charging on arc $(i, j)$ is performed only if trip $j$ is succeeded by trip $i$. Constraint $(10)$ restricts that charging on $\operatorname{arc}(i, j)$ can be performed only if time is available. Constraint (11) defines the upper bound of $W_{i j}$ on arc $(i, j) \in A$ according to the time available. Originally, constraint (11) is formulated as follows:

$$
W_{i j}+\left(Z_{i}-a_{j}-t_{i}-s_{i j}-t^{p}\right) r_{s} E_{i j} \leq 0,(i, j) \in A \text {. }
$$

To linearize quadratic terms $Z_{i} E_{i j}$, auxiliary variables $R_{i j}$ are introduced where $R_{i j}=Z_{i} E_{i j}$ is satisfied by constraints (14)-(17). Constraint (12) requires the battery SoC after charging not to exceed $u^{\max }$. Constraint (13) ensures that the charging time can only be integer times of the minimum charging time unit. Constraint (18) keeps the vehicle battery SoC within the safety range. Constraints (19)-(23) define the domains of the variables.

3.3. DP Equations to Define the Robustness of a Trip Chain. In this section, we introduce the DP equations to define the robustness of a trip chain under uncertainty set $U$. In graph 
$G$, a trip chain is represented by a path $P=\left\{o^{k}, v_{1}, v_{2}, \ldots, v_{n}, d^{k}\right\}$ starting from an operation start node $o^{k}$ to an operation end node $d^{k}$, visiting a sequence of trip nodes $v_{i}, i=1,2, \ldots, n$. In solving the RVRP, Agra et al. [29] showed that only the travel time vector $\tau \in \operatorname{ext}(U)$ needs to be considered in the problem formulation to ensure the robustness feasibility. $\operatorname{ext}(U)$ is the set that contains all the extreme points of set $U$. Because of the structure of set $U$, they defined DP recursive equations to define the robustness of a vehicle route. As our R-MD-EVSP problem shares a similar solution structure with the RVRP, we can define the robustness of a trip chain by the DP recursive equations (25) and (26) as follows.
Denote $Z_{i \gamma}$ as the earliest possible start time of node $v_{i}$ and $Y_{i \gamma}$ as the lowest battery SoC of the vehicle upon the start of $v_{i}$; let $W_{i j \gamma}$ and $\widetilde{W}_{i j \gamma}$ be the charging amount on $\operatorname{arc}(i, j)$, i.e., after the end of trip node $v_{i}$ and before the start of trip node $v_{j}$, when $\gamma \leq \Gamma^{t}$ trips from $v_{1}$ to $v_{i}$ are taking their maximum trip time, among which trip node $v_{i}$ takes the nominal trip time and maximum trip time, respectively. Denote $u_{i \gamma}^{\min }$ as the lower bound of $Y_{i \gamma}$ : if $\gamma<\Gamma^{t}$, $u_{i \gamma}^{\text {min }}=u^{\text {min }}+e_{i}+\widehat{e}_{i}$; otherwise, $u_{i \gamma}^{\text {min }}=u^{\text {min }}+e_{i}$.

$Z_{i \gamma}$ and $Y_{i \gamma}$ are defined by the recursive functions (25) and (26), respectively. A trip chain is robust if $Z_{i \gamma} \in\left[a_{i}, b_{i}\right]$ and $Y_{i \gamma} \in\left[u_{i \gamma}^{\min }, u^{\max }\right]$, for $\forall v_{i} \in P$ and $\gamma \leq \Gamma^{t}$. In fact, it suffices to check $Z_{i \Gamma^{t}}$ and $Y_{i \Gamma^{t}}$ for $v_{i} \in P$.

$$
Z_{j \gamma}=\left\{\begin{array}{l}
a_{0} \\
\max \left\{a_{j}, Z_{i \gamma}+t_{i}+t_{i j}\right\} \\
j=0 \\
Y_{j \gamma}= \begin{cases}\left.W_{j}, Z_{i \gamma}+t_{i}+\frac{W_{i j \gamma}}{r_{s}}+t_{i j}, Z_{i, \gamma-1}+t_{i}+\widehat{t}_{i}+\frac{\widetilde{W}_{i j \gamma}}{r_{s}}+t_{i j}\right\} \\
\min \left\{u^{\max }, Y_{i \gamma}-e_{i}+W_{i j \gamma}\right\}-e_{i j} & j=o \\
\min \left\{\min \left\{u^{\max }, Y_{i \gamma}-e_{i}+W_{i j \gamma}\right\}-e_{i j},\right. & \text { otherwise } \\
\left.\min \left\{u^{\max }, Y_{i, \gamma-1}-e_{i}-\widehat{e}_{i}+\widetilde{W}_{i j \gamma}\right\}-e_{i j}\right\} & \gamma=0\end{cases}
\end{array}\right.
$$

\subsubsection{The Optimal Total Charging Amount and Charging} Policy. Denote $H$ as the optimal total charging amount of a trip chain. $H$ is the maximum total charging amount required when the trip times varies within uncertainty set $U$ which is calculated as follows: let $b=\min \left\{n, \Gamma^{t}\right\}$ be the maximum number of trips of path $P$ that can attain their maximum trip time. Choose the $b$ trips with the maximum worst case trip time and take their maximum trip times. The rest of the trips take their nominal trip times. Then, $H$ is given by $H=u^{\min }+E-u^{\max }$ where $E$ is the total energy consumption of $P$. The charging policy for an e-bus carrying out trip chain $P$ is as follows: the e-buses can get recharged during the layover time between two successive trips for any flexible amount that is integer time of the unit charging time $t^{s}$ until the total charging amount reaches $H$.

3.4. Robust Problem Formulation. We introduce an MIP formulation for the R-MD-EVSP under cardinality uncertainty set $U$ by incorporating the DP equations for trip chain robustness checking into the deterministic model.
At each node $i \in V$, given $\gamma$ trips have taken their maximum trip time, $\gamma=0,1,2, \ldots, \Gamma^{t}$, variables $Z_{i \gamma}, Y_{i \gamma}$, $W_{i j \gamma}, \widetilde{W}_{i j \gamma}$ and parameter $u_{i \gamma}^{\min }$ are consistent with those defined in Section 3.3. Given that $\gamma$ trips on the trip chain from the first trip to trip $j$ (trip $j$ not included) are taking their maximum trip time, denote $E_{i j \gamma}$ and $\widetilde{E}_{i j \gamma}$ as the binary variables that are equal to 1 if vehicle charging can be carried out on arc $(i, j) \in A$ when trip $i$ takes its nominal and maximum trip time, respectively; denote $U_{i j \gamma}$ and $\widetilde{U}_{i j \gamma}$ as the integer variables that specify the number of time units an e-bus spends charging on arc $(i, j) \in A$ when trip $i$ takes its nominal and maximum trip time, respectively. Denote $R_{i j \gamma}$ and $\widetilde{R}_{i j \gamma}$ as the auxiliary variables defined to linearize the quadratic terms in the constraints.

In the robust model, the robustness of a trip chain is ensured by linearizing of the DP equations (25) and (26) and adding them into the problem constraints. The following constraints ((27)-(30)) are the linearized forms of the DP equations (25) and (26).

(i) Time consistency constraints: 


$$
\begin{array}{r}
Z_{j \gamma}-Z_{i \gamma}-t_{i}-s_{i j}+M\left(1-\sum_{k \in K} X_{i j k}\right) \geq 0, \quad(i, j) \in A_{p}, \gamma=0,1,2, \ldots, \Gamma^{t}, \\
Z_{j \gamma}-Z_{i, \gamma-1}-t_{i}-\widehat{t}_{i}-s_{i j}+M\left(1-\sum_{k \in K} X_{i j k}\right) \geq 0, \quad(i, j) \in A_{p}, \gamma=1,2, \ldots, \Gamma^{t} .
\end{array}
$$

Constraints (27) and (28) are the robust constraints corresponding to constraint (7). It is developed based on the linearization of equation (25), which means the time consistency of two successive trips should be satisfied for $\forall \gamma=0,1,2, \ldots, \Gamma^{t}$.

(ii) Battery SoC consistency constraints:

$$
\begin{aligned}
Y_{i \gamma}-e_{i}+W_{i j \gamma}-e_{i j}+M\left(1-\sum_{k \in K} X_{i j k}\right) \geq Y_{j \gamma}, \quad(i, j) \in A, \gamma=0,1,2, \ldots, \Gamma^{t}, \\
Y_{i, \gamma-1}-e_{i}-\widehat{e}_{i}+\widetilde{W}_{i j \gamma}-e_{i j}+M\left(1-\sum_{k \in K} X_{i j k}\right) \geq Y_{j \gamma}, \quad(i, j) \in A, \gamma=1,2, \ldots, \Gamma^{t} .
\end{aligned}
$$

Constraints (29) and (30) are the robust constraints corresponding to constraint (8). It is developed based on the linearization of equation (26), which means the battery SoC consistency of two successive trips should be satisfied for $\forall \gamma=0,1,2, \ldots, \Gamma^{t}$.

Other constraints are defined corresponding to the deterministic models with the variables substituted by the ones defined in the robust model. The R-MD-EVSP model is formulated as follows:

$$
\min \sum_{k \in K} \sum_{(i, j) \in A} c_{i j} X_{i j k}+\sum_{\gamma=0}^{\Gamma^{t}} \sum_{(i, j) \in A} g_{i j} W_{i j \gamma}+\sum_{\gamma=1}^{\Gamma^{t}} \sum_{(i, j) \in A} g_{i j} \widetilde{W}_{i j \gamma},
$$

s.t. constraints (2)-(5) and constraints (27)-(30).

$$
\begin{gathered}
a_{i} \leq Z_{i \gamma} \leq b_{i}, \quad i \in V, \gamma=0,1,2, \ldots, \Gamma^{t}, \\
E_{i j \gamma}-\sum_{k \in K} X_{i j k} \leq 0, \quad(i, j) \in A, \gamma=0,1,2, \ldots, \Gamma^{t}, \\
\widetilde{E}_{i j \gamma}-\sum_{k \in K} X_{i j k} \leq 0, \quad(i, j) \in A, \gamma=1,2, \ldots, \Gamma^{t}, \\
-\left(a_{j}-Z_{i \gamma}-t_{i}-s_{i j}-t^{p}\right)-M\left(1-E_{i j \gamma}\right) \leq 0, \quad(i, j) \in A, \gamma=0,1,2, \ldots, \Gamma^{t}, \\
-\left(a_{j}-Z_{i, \gamma-1}-t_{i}-\widehat{t}_{i}-s_{i j}-t^{p}\right)-M\left(1-\widetilde{E}_{i j \gamma}\right) \leq 0, \quad(i, j) \in A, \gamma=1,2, \ldots, \Gamma^{t}, \\
W_{i j \gamma}+R_{i j \gamma} r_{s}-\left(a_{j}-t_{i}-s_{i j}-t^{p}\right) r_{s} E_{i j \gamma} \leq 0, \quad(i, j) \in A, \gamma=0,1,2, \ldots, \Gamma^{t}, \\
\widetilde{W}_{i j \gamma}+\widetilde{R}_{i j \gamma} r_{s}-\left(a_{j}-t_{i}-\widehat{t}_{i}-s_{i j}-t^{p}\right) r_{s} \widetilde{E}_{i j \gamma} \leq 0, \quad(i, j) \in A, \gamma=1,2, \ldots, \Gamma^{t}, \\
W_{i j \gamma}+Y_{i \gamma}-e_{i}-u^{\max } \leq 0, \quad(i, j) \in A, \gamma=0,1,2, \ldots, \Gamma^{t}, \\
\widetilde{W}_{i j \gamma}+Y_{i, \gamma-1}-e_{i}-\widehat{e}_{i}-u^{\max } \leq 0, \quad(i, j) \in A, \gamma=1,2, \ldots, \Gamma^{t}, \\
W_{i j}-r_{s} t^{s} U_{i j \gamma}=0, \quad(i, j) \in A, \gamma=0,1,2, \ldots, \Gamma^{t},
\end{gathered}
$$




$$
\begin{aligned}
& W_{i j}-r_{s} t^{s} \widetilde{U}_{i j \gamma}=0, \quad(i, j) \in A, \gamma=0,1,2, \ldots, \Gamma^{t}, \\
& R_{i j \gamma}-b_{i} E_{i j \gamma} \leq 0, \quad(i, j) \in A, \gamma=0,1,2, \ldots, \Gamma^{t}, \\
& a_{i} E_{i j \gamma}-R_{i j \gamma} \leq 0, \quad(i, j) \in A, \gamma=0,1,2, \ldots, \Gamma^{t}, \\
& R_{i j \gamma}-Z_{i \gamma}+a_{i}\left(1-E_{i j \gamma}\right) \leq 0, \quad(i, j) \in A, \gamma=0,1,2, \ldots, \Gamma^{t}, \\
& Z_{i \gamma}-b_{i}\left(1-E_{i j \gamma}\right)-R_{i j \gamma} \leq 0, \quad(i, j) \in A_{p}, \gamma=0,1,2, \ldots, \Gamma^{t}, \\
& \widetilde{R}_{i j \gamma}-b_{i} \widetilde{E}_{i j \gamma} \leq 0, \quad(i, j) \in A, \gamma=1,2, \ldots, \Gamma^{t}, \\
& a_{i} \widetilde{E}_{i j \gamma}-\widetilde{R}_{i j \gamma} \leq 0, \quad(i, j) \in A, \gamma=1,2, \ldots, \Gamma^{t}, \\
& \widetilde{R}_{i j \gamma}-Z_{i, \gamma-1}+a_{i}\left(1-\widetilde{E}_{i j \gamma}\right) \leq 0, \quad(i, j) \in A, \gamma=1,2, \ldots, \Gamma^{t}, \\
& Z_{i, \gamma-1}-b_{i}\left(1-\widetilde{E}_{i j \gamma}\right)-\widetilde{R}_{i j \gamma} \leq 0, \quad(i, j) \in A, \gamma=1,2, \ldots, \Gamma^{t}, \\
& u_{i \gamma}^{\min } \leq Y_{i \gamma} \leq u^{\max }, \quad i \in V, \gamma=0,1,2, \ldots, \Gamma^{t}, \\
& W_{i j \gamma} \geq 0, \quad(i, j) \in A, \gamma=0,1,2, \ldots, \Gamma^{t}, \\
& \widetilde{W}_{i j \gamma} \geq 0, \quad(i, j) \in A, \gamma=0,1,2, \ldots, \Gamma^{t}, \\
& X_{i j k} \in\{0,1\}, \quad(i, j) \in A, k \in K, \\
& E_{i j \gamma} \in\{0,1\}, \quad(i, j) \in A, \gamma=0,1,2, \ldots, \Gamma^{t}, \\
& R_{i j \gamma} \geq 0, \quad(i, j) \in A, \gamma=0,1,2, \ldots, \Gamma^{t}, \\
& U_{i j \gamma} \in Z, \quad(i, j) \in A, \gamma=0,1,2, \ldots, \Gamma^{t}, \\
& \widetilde{E}_{i j \gamma} \in\{0,1\}, \quad(i, j) \in A, \gamma=0,1,2, \ldots, \Gamma^{t}, \\
& \widetilde{R}_{i j \gamma} \geq 0, \quad(i, j) \in A, \gamma=0,1,2, \ldots, \Gamma^{t}, \\
& \widetilde{U}_{i j \gamma} \in Z, \quad(i, j) \in A, \gamma=0,1,2, \ldots, \Gamma^{t} .
\end{aligned}
$$

Objective (31) consists of the vehicle usage, empty travel, and the sum of charging cost in all possible cases regarding the realizations of the trip times. The worst-case charging cost cannot be expressed explicitly; therefore, it is not included in the objective but calculated after the optimal solution is obtained. Constraint (32) limits the departure time of each trip to be within the departure time window.
Constraints (33) and (34) require that charging on arc $(i, j)$ is performed only if trip $j$ is succeeded by trip $i$. Constraints (35) and (36) restrict that charging on $\operatorname{arc}(i, j)$ can be performed if time is available. Constraints (37) and (38) define the upper bound of $W_{i j}$ on arc $(i, j) \in A$ according to the available time. Originally, constraints (37) and (38) are formulated as follows:

$$
\begin{aligned}
W_{i j \gamma}+\left(Z_{i \gamma}-a_{j}-t_{i}-s_{i j}-t^{p}\right) r_{s} E_{i j \gamma} \leq 0, \quad(i, j) \in A, \gamma=0,1,2, \ldots, \Gamma^{t}, \\
\widetilde{W}_{i j \gamma}+\left(Z_{i, \gamma-1}-a_{j}-t_{i}-\widehat{t}_{i}-s_{i j}-t^{p}\right) r_{s} \widetilde{E}_{i j \gamma} \leq 0, \quad(i, j) \in A, \gamma=1,2, \ldots, \Gamma^{t} .
\end{aligned}
$$


To linearize quadratic terms $Z_{i \gamma} E_{i j \gamma}$ and $Z_{i, \gamma-1} \widetilde{E}_{i j \gamma}$, auxiliary variables $R_{i j \gamma}$ and $\widetilde{R}_{i j \gamma}$ are introduced where $R_{i j \gamma}=$ $Z_{i \gamma} E_{i j \gamma}$ is satisfied by constraints (43)-(46) and $\widetilde{R}_{i j \gamma}=Z_{i, \gamma-1} \widetilde{E}_{i j \gamma}$ is satisfied by constraints (47)-(50).

Constraints (39) and (40) require the battery SoC after charging not to exceed $u^{\max }$. Constraints (41) and (42) ensure that the charging time can only be integer times of the minimum charging time unit. Constraint (49) keeps the vehicle battery SoC within the safety range. Constraints (52)-(60) define the domains of the variables.

\section{Branch-and-Price Method}

In this section, we introduce a $\mathrm{BP}$ algorithm to solve the R-MD-EVSP based on the set partitioning formulation. Let $\Omega$ be the set of feasible trip chains. Each trip chain $p \in \Omega$ is defined by $c_{p}$, the total operation cost, and $a_{p i}, i \in T$, a binary parameter that is equal to 1 if trip $i$ is carried out in $p$. Denote $\theta_{p}, p \in \Omega$, as a binary variable that takes value 1 if trip chain $p$ is selected to be part of the solution. The set partitioning formulation of the R-MD-EVSP is as follows:

$$
\begin{aligned}
& \min \sum_{p \in \Omega} c_{p} \theta_{p}, \\
& \text { s.t. } \sum_{k \in K} a_{p i} \theta_{p}=1, \quad i \in T, \\
& \quad \theta_{p} \in\{0,1\}, \quad p \in \Omega .
\end{aligned}
$$

Objective (63) minimizes the total operation cost of the schedule. Constraint (64) ensures that each trip is carried out once by a vehicle. As set $\Omega$ includes a large number of feasible trip chains, it is impractical to solve the model directly. Therefore, we developed a column generation (CG) algorithm to solve the linear relaxation of models (63)-(65) called master problem (MP). The CG algorithm starts by solving the linear relaxation of models (63)-(65) with an initial set of feasible trip chains $\bar{\Omega}$, called the restricted master problem (RMP). In each iteration, a pricing problem is solved to generate columns with negative reduced cost to be added to the RMP. Let $\pi_{i}, i \in T$, be the dual variables associated with constraint (64). Let $\bar{c}_{p}$ be the reduced cost of trip chain $p \in \Omega$ with respect to $\pi_{i}, i \in T$, i.e., $\bar{c}_{p}=c_{p}-\sum_{i \in T} a_{p i} \pi_{i}$. The pricing problem is defined as follows:

$$
\min \sum_{p \in \Omega} \bar{c}_{p} \theta_{p}
$$

A feasible trip chain $p \in \Omega$ corresponds to an $o$ - $d$ path in $G$. The problem aims at finding a feasible trip chain with the minimum reduced cost. We modify the cost of each arc $(i, j) \in A$ by a modified cost $\bar{c}_{i j}=c_{i j}-\pi_{i}$ where $\pi_{i}=0$ for $i \in\{o, d\}$. The reduced cost of an $o$ - $d$ path $p$ equals the sum of $\bar{c}_{i j},(i, j) \in p$. The problem is a resource-constrained elementary shortest path problem (RCESPP), and we solve it by developing a label setting algorithm [35]. The CG alternates between the optimization of the RMP and the pricing problems until no more columns with negative reduced cost are generated, implying that the MP has been solved to optimality.

4.1. Robustness Checking of a Trip Chain. In solving the pricing problem, we need to generate robust $o$ - $d$ paths in graph $G$. Based on the DP equations (25) and (26) defined in Section 3.3 , the robustness of path $P$ can be checked by the following procedure.

The Robustness Checking Procedure. On an arc $\left(v_{i}, v_{j}\right)$ of path $P$, for each $\gamma \leq \Gamma^{t}$, determine the values of $W_{i j \gamma}$ and $\widetilde{W}_{i j \gamma}$ by equations (67) and (68). Then, the values of $Z_{j \gamma}$ and $Y_{j \gamma}$ are determined by equations (25) and (26), respectively. Path $P$ is robustness feasible if $Z_{i \gamma} \in\left[a_{i}, b_{i}\right]$ and $Y_{i \gamma} \in\left[u_{i \gamma}^{\min }, u^{\max }\right]$ for $v_{i} \in P$ and $\gamma \leq \Gamma^{t}$.

$$
W_{i j \gamma}=\max \left\{0, \min \left\{u^{\max },\left(a_{j}-Z_{i \gamma}-t_{i}-t_{i j}-t^{p}\right) r_{s}\right\}\right\},
$$

$$
\widetilde{W}_{i j \gamma}=\max \left\{0, \min \left\{u^{\max },\left(a_{j}-Z_{i, \gamma-1}-t_{i}-\widehat{t}_{i}-t_{i j}-t^{p}\right) r_{s}\right\}\right\} .
$$

4.2. Label Setting for the Pricing Problem. In this section, we describe the label setting algorithm for generating robust $o-d$ paths based on the robustness checking approach proposed in Section 4.1. In solving the RCESPP on graph $G$, the labels are used to represent the partial paths starting from the start node and the resources accumulated along the path. New labels are generated by extending the existing labels on graph $G$ following the resource extension functions (REFs). New labels are checked for resource feasibility and infeasible labels are discarded. At each node, dominance rule is then applied to eliminate the labels which cannot lead to a path with a better reduced cost than the dominate labels.

Denote $L_{i}=\left(C_{i}, R_{i \gamma}^{t}, R_{i \gamma}^{e}\right), \gamma \leq \Gamma^{t}$, as a label representing a partial path from the start node $o$ to a node $i \in V$ with three kinds of resource defined as follows: $C_{i}$ is the reduced cost of the path, $R_{i \gamma}^{t}$ is the earliest departure time of node $i$, and $R_{i \gamma}^{e}$ is the lowest battery SoC of the vehicle upon the start of trip $i$, when $\gamma$ trips from node $o$ to node $i$ are attaining their maximum trip time.

In the VRP, when the customer time windows are wide, two customers can be served in different orders so that the graph is cyclic. As such, in solving the pricing problem, at a given node $i$ in the graph, an elementary resource is defined for each customer to record the number of times the customer has been visited on the partial path $o, 1,2, \ldots, i[31]$. In the R-MD-EVSP, because the departure time window of the trips is small, any two trips cannot be served in different orders and graph $G$ is acyclic. Therefore, in solving the pricing problem, the elementary resource is not needed.

Based on the recursive equations (25) and (26) and the feasibility checking approach introduced in Section 4.1, the forward extension of a label $L_{i}$ to a label $L_{j}$ along an arc $(i, j) \in A$ is performed by REFs (69)-(71). As the feasibility checking approach always ensures $R_{j \gamma}^{e} \in\left[u_{j \gamma}^{\min }, u^{\max }\right]$, the 
resulting label $L_{j}$ is robust feasible if and only if $R_{j \gamma}^{t} \in\left[a_{j}, b_{j}\right], \gamma=0,1,2, \ldots, \Gamma^{t}$.

$$
\begin{aligned}
& C_{j}=C_{i}+c_{i j}-\pi_{i}, \\
& R_{j \gamma}^{e}=\min \left\{u^{\max }, R_{i \gamma}^{e}-e_{i}+W_{i j \gamma}-e_{i j}, R_{i, \gamma-1}^{e}-e_{i}-\widehat{e}_{i}+\widetilde{W}_{i j \gamma}-e_{i j}\right\}, \\
& R_{j \gamma}^{t}=\max \left\{a_{i}, R_{i \gamma}^{t}+t_{i}+\frac{W_{i j \gamma}}{r_{s}}+t_{i j}, R_{i \gamma-1}^{t}+t_{i}+\widehat{t}_{i}+\frac{\widetilde{W}_{i j \gamma}}{r_{s}}+t_{i j}\right\} .
\end{aligned}
$$

Variables $W_{i j \gamma}$ and $\widetilde{W}_{i j \gamma}$ are consistent with those defined in Section 3.3. $c_{i j}$ is the estimated cost on arc $(i, j) \in A$ consisting of the cost of vehicle usage, empty travel, trip start delay, and charging cost. $c_{i j}$ is computed as follows. Let $P_{j}=\{o, 1,2, \ldots, i, j\}$ be a partial path from node o to node $j$. Denote $H_{j}$ as the maximum total charging amount required for $P_{j}$ when the trip times varies within uncertainty set $U \cdot c_{i j}^{\prime}=c_{i j}+g_{i j}\left(H_{j}-H_{i}\right)$ where $c_{i j}$ is the vehicle usage and empty travel cost assigned to $\operatorname{arc}(i, j)$; $g_{i j}\left(H_{j}-H_{i}\right)$ is the increased charging cost.

In the label setting algorithm, dominance rule plays a critical role in reducing the number of extensions required as the labeling process goes on. In Proposition 1, we introduce a dominance rule for our label setting algorithm to improve the computational efficiency.

Proposition 1. Let $L_{i}^{1}$ and $L_{i}^{2}$ be the two labels associated with the paths ending at node $i$. $L_{i}^{1}$ dominates $L_{i}^{2}$ if (i) $C_{i}^{1} \leq C_{i}^{2}$; (ii) $R_{i \gamma}^{e 1} \geq R_{i \gamma}^{e 2}, \quad \gamma=0,1,2, \ldots, \Gamma^{t}$; and (iii) $R_{i \gamma}^{t 1} \leq R_{i \gamma}^{t 2}$, $\gamma=0,1,2, \ldots, \Gamma^{t}$.

4.3. Heuristic Pricing. The label setting algorithm can be time-consuming, especially in solving large-scale instances. As we know, it is not necessary to solve the pricing problem to its optimal and return a column with the most negative reduced cost at each iteration of the CG. The computational efficiency of the GC procedure can be improved by only finding suboptimal solutions in the pricing problem at the expense of an increase in the number of iterations. As such, we adopt heuristic decisions before invoking the exact pricing algorithm. Instead of storing all the nondominant columns in the label setting, we maintain only a prespecified number of them to improve the algorithm efficiency. When a new nondominant label is added to the list and the total number of labels has reached the limit, the label with the largest objective value in the list will be discarded. In this way, fewer labels are extended at each node, speeding up the process of negative path detection. Note that this heuristic may lead to more CG iterations and the number of labels maintained should be carefully set through preliminary experiment.

4.4. Branching. An initial solution is formulated by assigning each e-bus with one trip. After each CG procedure ends, to obtain the integer solution, branches are created in the branch-and-bound tree by branching on the total flow of the arcs $\left(x_{i j}\right)_{(i, j) \in A}$. If not all the $\left(x_{i j}\right)_{(i, j) \in A}$ are integers, we choose to branch on $x_{i j}$ that has the highest fractional value, creating two nodes, one with $x_{i j}=1$ and the other with $x_{i j}=0$. Otherwise, an integer solution is obtained. A depthfirst search strategy is used to explore the branch-and-bound tree. In order to obtain integer solutions more efficiently, after each CG procedure ends, $x_{i j}$ values in the RMP that are larger than 0.99 are set to 1 , while $x_{i j}$ values that are less than 0.01 are set to 0 . The preliminary experiments show that this setting works well on speeding up the computational time and has minor impact on the solution quality.

4.5. Obtaining Optimal Charging Plan. After the optimal schedule is obtained by the BP algorithm, we further generate the optimal charging plan for each individual trip chain, considering the time-of-use tariff. The problem can be defined as a robust fixed-route electric vehicle refueling problem (R-FRVRP) which aims to determine the optimal charging time and amount for an e-bus on a given trip chain.

\section{Numerical Experiments}

We conducted numerical experiments based on the case of bus routes in Shenzhen. We first conducted experiments on single-route scheduling and then on multiroute scheduling where e-buses are allowed to carry out trips on different routes. In the following cases, plug-in DC chargers are established in the depots, the majority of which have the maximum charging power of $100 \mathrm{~kW}$. BYD e-buses with a battery capacity of $260 \mathrm{kWh}$, length of $10.5 \mathrm{~m}$, weight of $18000 \mathrm{~kg}$, and maximum driving distance of $220 \mathrm{~km}$ operate on the routes. The characteristics of the bus routes and charging facilities are presented in Table 2. According to the historical running data, the charging rate is set to be $1.6 \mathrm{kWh}$ per minute, and the battery energy consumption rate is estimated to be $0.6 \mathrm{kWh}$ per minute. As defined in Section 3.1 , the energy consumption of a trip depends on the trip time following the equation $\delta_{i}^{e}=\alpha \delta_{i}^{t} r_{u}$. Here we set $\alpha=1$. The charging preparation time $t^{p}$ and minimum charging time $t^{s}$ are set as $2 \mathrm{~min}$ and $5 \mathrm{~min}$, respectively. Parameter $\alpha$ takes the value of 0.5 . The daily usage cost of an e-bus $c^{f}$ is set as $1000 \mathrm{CNY}$. Figure 3 shows the time-of-use tariff in Shenzhen, which is used to set the values of $g_{i j},(i, j) \in A$. 
TABLE 2: Characteristics of the bus routes in the single and multiroute scheduling cases.

\begin{tabular}{|c|c|c|c|c|c|c|c|c|}
\hline Route & $\begin{array}{l}\text { Length } \\
(\mathrm{km})\end{array}$ & $\begin{array}{l}\text { Number of } \\
\text { stops }\end{array}$ & $\begin{array}{l}\text { Operation } \\
\text { period }\end{array}$ & $\begin{array}{l}\text { Headway } \\
\text { (min) }\end{array}$ & $\begin{array}{l}\text { Ave. trip time } \\
(\text { min) }\end{array}$ & $\begin{array}{c}\text { Number of } \\
\text { trips }\end{array}$ & $\begin{array}{c}\text { End depot } \\
\text { I }\end{array}$ & $\begin{array}{c}\text { End depot } \\
\text { II }\end{array}$ \\
\hline M133 & 37.5 & 44 & $6: 10-22: 30$ & 5-12 & 110 & 276 & $1(16)$ & $2(8)$ \\
\hline E7 & 39.7 & 15 & $7: 00-22: 00$ & $10-60$ & 70 & 84 & $3(20)$ & $4(5)$ \\
\hline E11 & 75.5 & 18 & $6: 00-21: 30$ & $6-10$ & 120 & 194 & 3 & $5(10)$ \\
\hline E14 & 41.9 & 22 & $7: 30-22: 00$ & $15-50$ & 110 & 70 & 3 & $6(10)$ \\
\hline E22 & 57.1 & 19 & $6: 30-20: 00$ & 60 & 120 & 34 & 3 & $7(5)$ \\
\hline 42 & 17.3 & 23 & $6: 30-22: 30$ & $10-15$ & 45 & 132 & $8(10)$ & $9(8)$ \\
\hline 43 & 17.6 & 25 & $6: 30-22: 30$ & $10-15$ & 60 & 130 & 3 & 9 \\
\hline 81 & 31.6 & 44 & $6: 30-21: 30$ & $5-15$ & 80 & 204 & 3 & 8 \\
\hline
\end{tabular}

Note. End depots are numbered from 1 to 9 as follows: 1, Changlingdong depot; 2, Shekou bus depot; 3, Shenzhen North Railway Station; 4, Longgangyiwu bus depot; 5, Nanaoxinda bus depot; 6, Tantou West; 7, Hangzi bus depot; 8, Moon Bay; 9, Window of the World. The values in the brackets next to the depot index are the number of charging piles established at the depots.

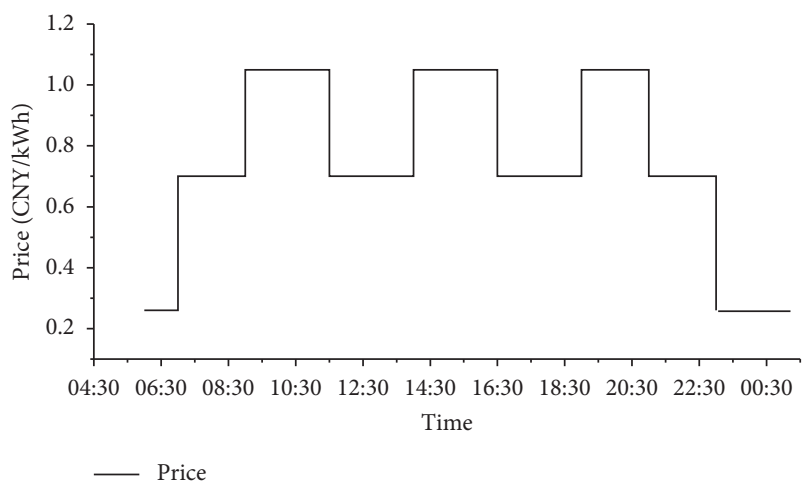

Figure 3: Time-of-use tariff in Shenzhen.

The MIP model was solved by the standard optimization solver Cplex 12.6, and the BP framework was coded in Java using Cplex 12.6 to solve the RMP. All the experiments were run on a PC with Windows 10, Intel Core i5-8250U, $1.80 \mathrm{GHz}$, and $8 \mathrm{~GB}$ RAM.

5.1. Single-Route Scheduling. We first analyze the performance of the MIP model and BP algorithm based on Route M133 in Shenzhen. A display of bus Route M133 is shown in Figure 4.

In generating a timetable, estimation of the scheduled trip times is critical. We adopted the method suggested in [27] to generate homogeneous running time (HRT) periods, the trip time within each of which follows the same distribution. We generate the HRT periods for Route M133 based on the trip time data of 100 weekdays from August 1 to December 31 in 2017. Figure 5 presents the distribution of the trip time of Route M133 during different periods of the day. Within each HRT period, the scheduled trip time is set according to a rule-of-thumb: the average trip time plus the standard deviation of the trip time. Figure 6 shows the scheduled trip time and maximum deviation from the scheduled trip time within each running time period. We generate the timetable including 276 trips. Except for the original timetable, we also create timetables with the number of scheduled trips ranging from 64 to 154 by changing the headway requirements. These timetables account for the small-scale instances.
5.1.1. Results. The performance of the MIP model and BP algorithm with uncertainty budget $\Gamma^{t}=0,1,2,3$ is presented in Table 3. The deterministic solution corresponds to the case with $\Gamma^{t}=0$. The table shows the objective of the MIP model obtained by Cplex and BP algorithm (Obj), number of vehicles used $(\# \mathrm{~V})$ in the solution obtained by the $\mathrm{BP}$, optimal gap of the MIP and BP, and the computational time. When the MIP gap is higher than $90 \%$, we report the root relaxation solution obtained by Cplex in the bracket. If the root relaxation is not solved by Cplex within $3600 \mathrm{~s}$, an em dash ("-") is used.

The results indicate that Cplex is not able to solve the model to near optimal in a short time. Some instances are not solved by Cplex in $3600 \mathrm{~s}$; however, the BP algorithm is able to generate high-quality solutions with small BP gaps within a reasonable computational time. The objective obtained by the BP algorithm is consistently lower than that obtained by the MIP model. For a given instance, the total operational cost, including the cost of vehicle usage and charging, becomes higher with the increase of the uncertainty budget $\Gamma^{t}$. This is due to the more vehicles put into use and a larger amount of planned daytime charging amount. When the level of protection $\Gamma^{t}$ increases, the planned daytime charging amount becomes higher if the number of vehicles used stays the same; the daytime charging amount can be reduced when the number of vehicles used increases.

5.1.2. Impact of the Trip Start Buffer Time. We investigated the impact of trip start buffer time on the optimal schedule. Each case is named as "number of trips-budget value." The trip start delay time $t^{d}$ varies among $0,3,5$, and 8 minutes. $t^{d}=0$ means that trip start delay is not permitted. As shown in Figure 7, the number of e-buses used is reduced with the increase of $t^{d}$ in cases "96-0," "112-0," and "276-0." The results indicate that allowing for trip start buffer time can reduce the number of e-buses put into operation in some cases. Trip start buffer time can increase time flexibility of the schedule and saves the operational cost in some scenarios. With the increase of the uncertainty budget, the planned charging amount increases, protecting the schedule against the variation of energy consumption. 


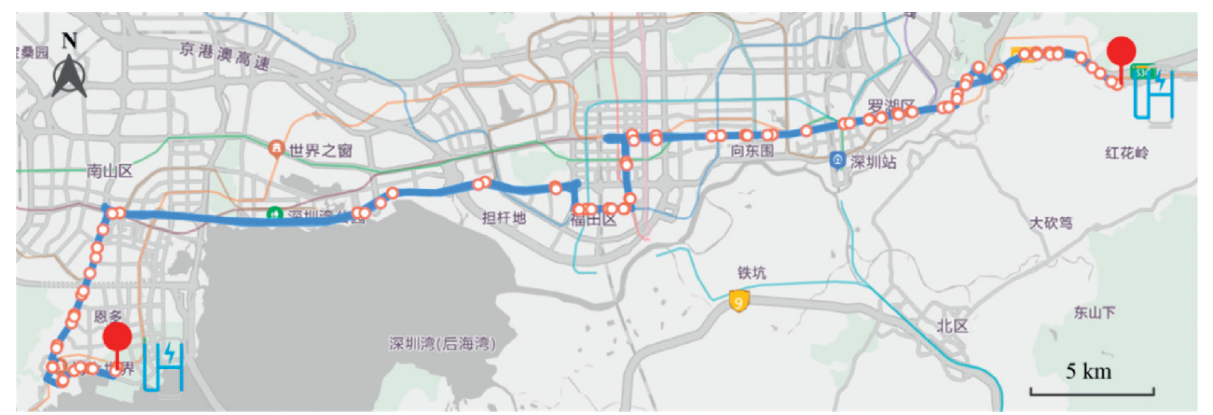

FIgUre 4: A display of bus Route M133 in Shenzhen.

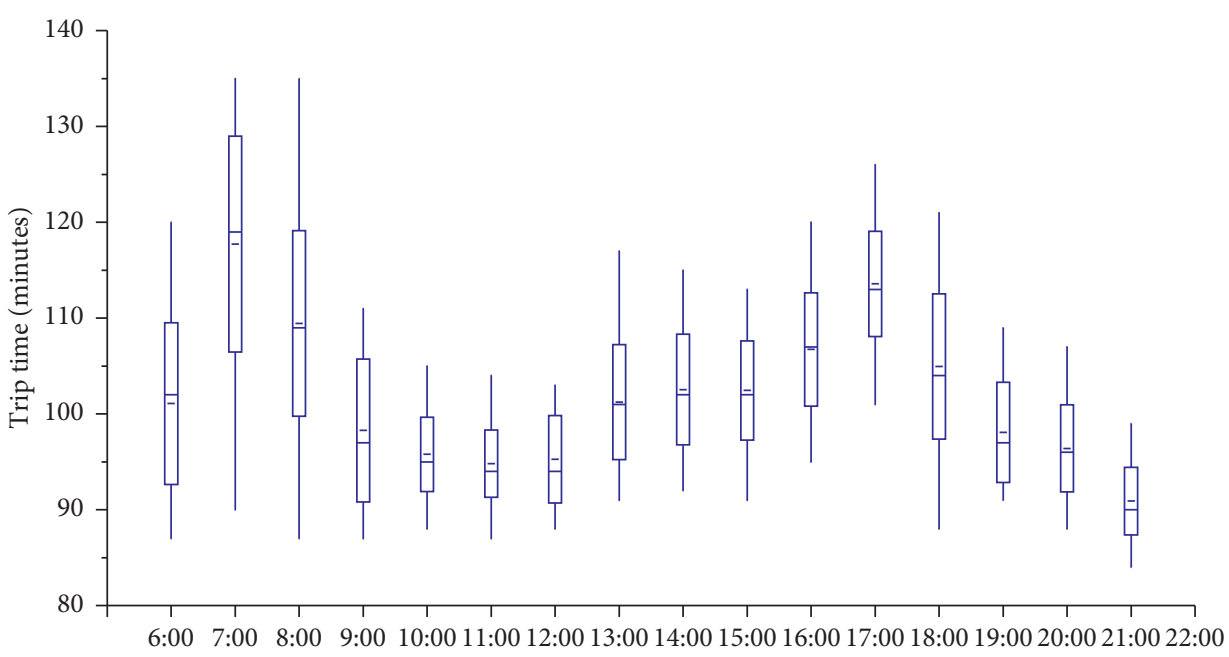

Figure 5: Trip time distribution of Route M133 in a day based on the historical running data.

5.2. Multiroute Scheduling. We carried out numerical experiments on two cases with multiple bus routes and depots. The multiroute scheduling is applied in the cases. The characteristics and layouts of the transit system in the case are shown in Table 2 and Figure 8. Case I includes five depots and four express bus routes: Route Nos. E7, E11, E14, and E22, that all have one end stop at the Shenzhen North Railway Station, formulating a hub-and-spoke topology. The timetable of the four routes includes a total number of 382 trips. Case II includes three depots and three bus routes: Route Nos. 42, 42, and 81, that share end stops with each other, formulating a circle topology. All the depots are equipped with charging facilities. The timetable of case I and case II includes a total number of 382 and 466 trips.

5.2.1. Results. We obtained the optimal schedule for cases I and II by the BP algorithm. The computational results are shown in Table 4. The table shows the objective of the MIP model obtained by the BP algorithm (Obj), number of vehicles used $(\# \mathrm{~V})$, total charging amount, computational time, and BP gap with uncertainty budget $\Gamma^{t}=0,1,2,3$.

5.3. Schedule Robustness. We investigated the schedule robustness through Monte Carlo simulation based on the randomly generated trip time data. The simulation is performed by generating $N^{\text {ran }} d=10000$ random realizations of trip time from normal distribution $N\left(t_{i}^{a}, s d_{i}\right)$ for all trip nodes $i \in T$, where $t_{i}^{a}$ and $s d_{i}$ are the mean and standard deviation of the trip time. Each random realization represents one day's e-bus schedule.

Table 5 presents the statistics on the deterministic $\left(\Gamma^{t}=0\right)$ and robust $\left(\Gamma^{t}=1,2,3\right)$ solutions for single-route instances. The number of vehicles used $(\# \mathrm{~V})$, infeasibility rate of the trip chain (IR-TC), schedule (IR-S), and price of robustness (PoR) are reported. Denote the total number of infeasible trip chains as $N_{t c}^{I F}$. If the schedule contains infeasible trip chains, the schedule is regarded as infeasible. Denote the total number of infeasible schedules as $N_{s}^{I F}$. IRTC is calculated as $N_{t c}^{I F} /\left(N^{\text {rand }} * N^{v e h}\right)$ where $N^{v e h}$ is the number of e-buses used; IR-S is calculated as $N_{s}^{I F} / N^{\text {rand }}$. PoR is the percentage of increase in the operational cost of the robust solutions compared with that of the deterministic solution.

About $0.94 \%$ to $9.81 \%$ of the simulated e-bus trip chains experience delays using the deterministic solutions. With $\Gamma^{t}=1$, the infeasibility rate drops to almost zero while the total operational cost increases by $1.25 \%$ to $16.14 \%$. We calculated the penalty of the trips chains incurred by the violation of the trip start time window and battery SoC safety 


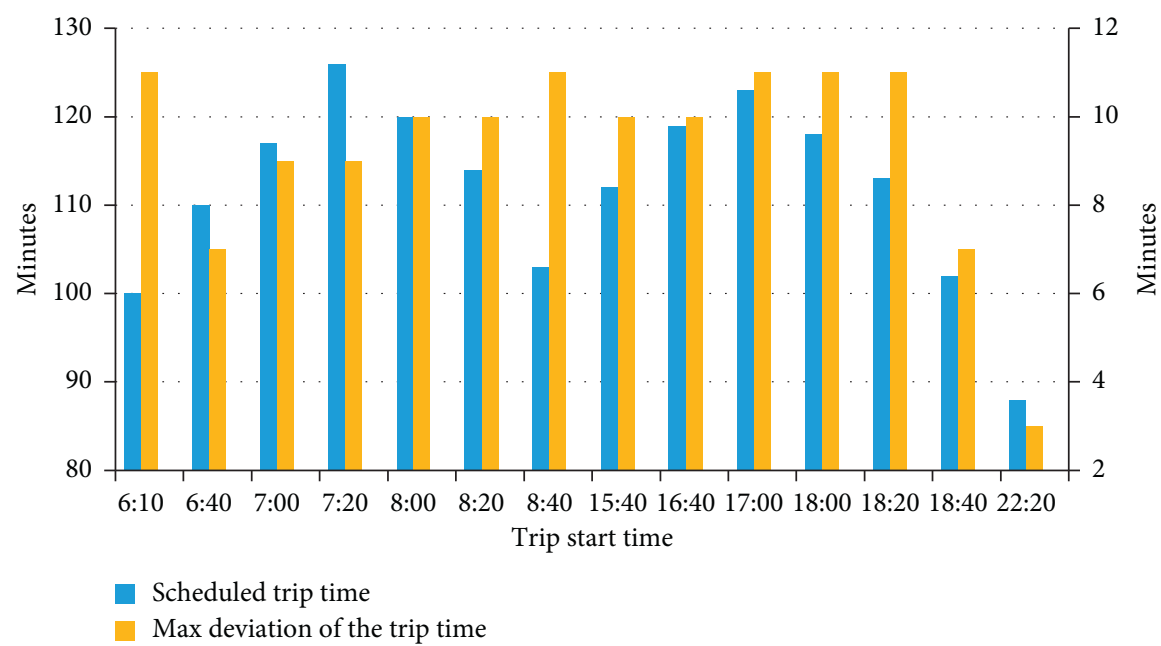

(a)

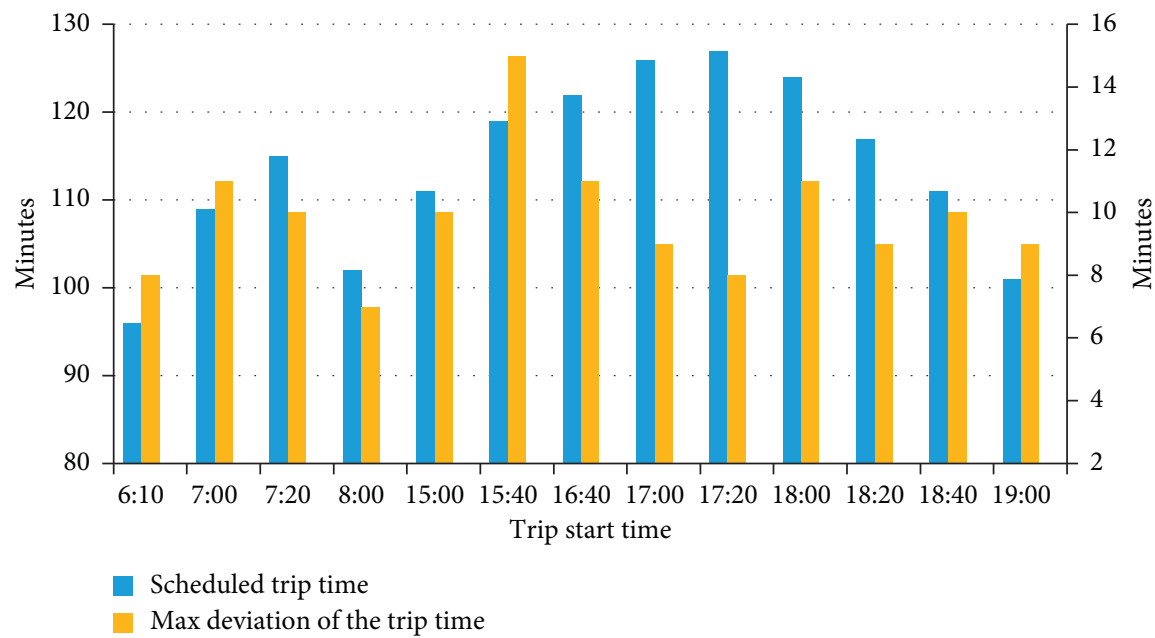

(b)

FIgURE 6: HRT periods of Route M133 generated based on the historical running data. (a) Outbound. (b) Inbound.

TABLE 3: Computational results of the MIP model and BP on single-route instances.

\begin{tabular}{|c|c|c|c|c|c|c|c|c|}
\hline \multirow{2}{*}{ Number of trips } & \multirow{2}{*}{$\Gamma^{t}$} & \multicolumn{2}{|c|}{ Obj } & \multirow{2}{*}{$\begin{array}{l}\# \mathrm{~V} \\
\mathrm{BP}\end{array}$} & \multicolumn{2}{|c|}{ Gap (\%) } & \multicolumn{2}{|c|}{ Run time (s) } \\
\hline & & MIP & $\mathrm{BP}$ & & MIP & $\mathrm{BP}$ & Cplex & $\mathrm{BP}$ \\
\hline \multirow[t]{4}{*}{62} & 0 & 16916.5 & 16912.8 & 16 & 5.4 & 0 & 3600 & 0 \\
\hline & 1 & 18107.3 & 16400.0 & 16 & 11.64 & 0 & 3600 & 1 \\
\hline & 2 & 19876.8 & 16800.0 & 16 & 19.50 & 0 & 3600 & 1 \\
\hline & 3 & 21763.3 & 17000.0 & 17 & 26.48 & 0.12 & 3600 & 1 \\
\hline \multirow[t]{4}{*}{96} & 0 & 22900.7 & 22676.7 & 21 & 8.30 & 0.14 & 3600 & 8 \\
\hline & 1 & 25664.7 & 25504.5 & 22 & 18.18 & 0.21 & 3600 & 5 \\
\hline & 2 & 30395.0 & 27279.2 & 22 & 30.91 & 0.21 & 3600 & 6 \\
\hline & 3 & 35098.3 & 33331.9 & 22 & 40.17 & 0.26 & 3600 & 8 \\
\hline \multirow[t]{4}{*}{112} & 0 & 26294.3 & 25711.1 & 23 & 12.53 & 0.42 & 3600 & 10 \\
\hline & 1 & 30508.7 & 26714.7 & 24 & 24.61 & 0.60 & 3600 & 7 \\
\hline & 2 & 36842.2 & 30202.2 & 24 & 37.57 & 0.58 & 3600 & 8 \\
\hline & 3 & 51072.5 & 33858.5 & 24 & 54.97 & 0.71 & 3600 & 10 \\
\hline \multirow[t]{4}{*}{154} & 0 & 38082.3 & 36578.7 & 33 & 13.35 & 0.20 & 3600 & 24 \\
\hline & 1 & 69344.3 & 37041.8 & 33 & 52.41 & 0.22 & 3600 & 34 \\
\hline & 2 & $(33000)$ & 42180.0 & 33 & - & 0.68 & - & 56 \\
\hline & 3 & $(33000)$ & 45606.0 & 33 & - & 0.84 & - & 13 \\
\hline \multirow[t]{4}{*}{276} & 0 & $(49000)$ & 56443.3 & 49 & - & 0.82 & - & 129 \\
\hline & 1 & $(49000)$ & 67309.1 & 49 & - & 0.86 & - & 169 \\
\hline & 2 & - & 82386.7 & 49 & - & 1.02 & - & 191 \\
\hline & 3 & - & 99134.4 & 49 & - & 0.92 & - & 113 \\
\hline
\end{tabular}



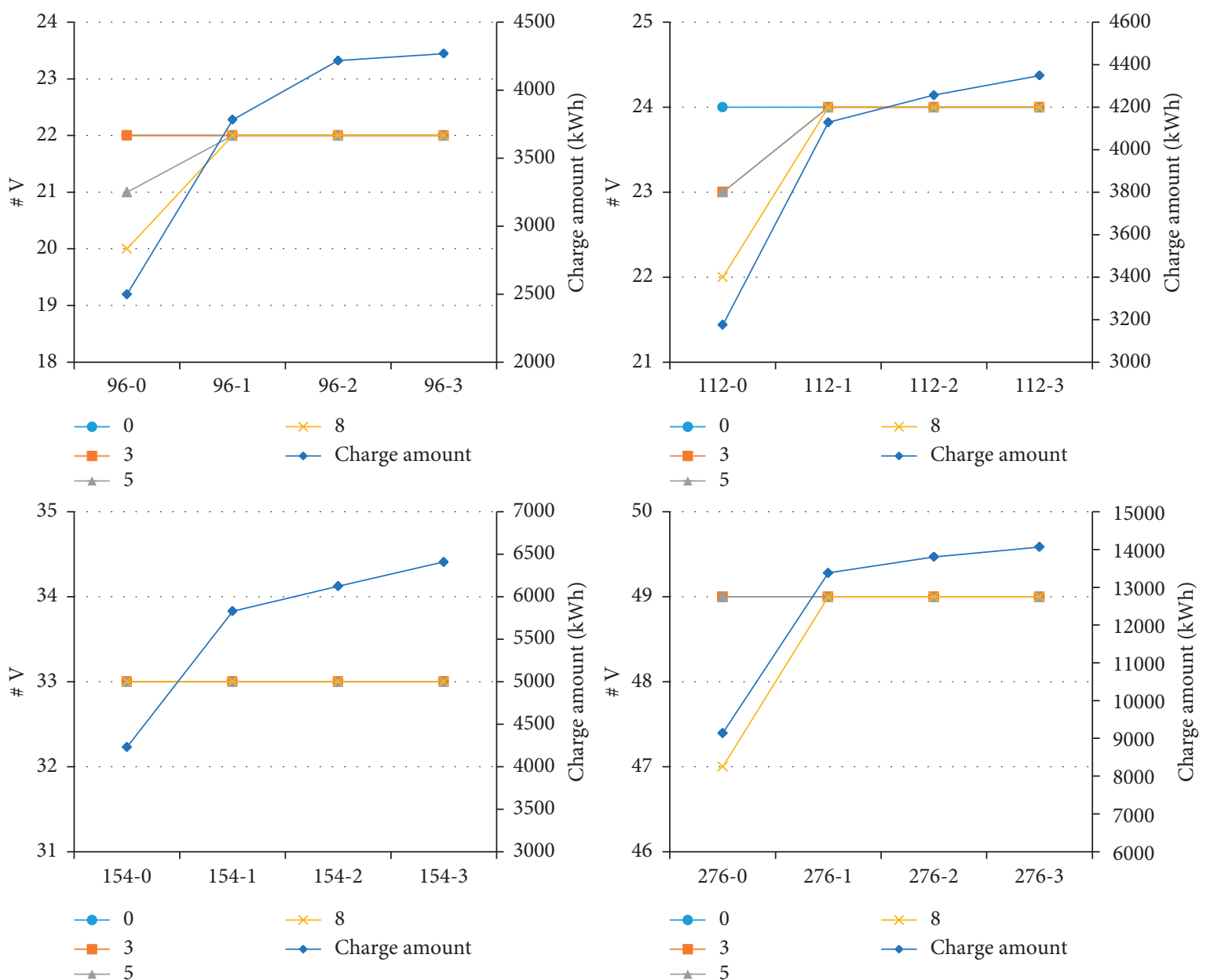

FIgURE 7: The influence of departure delay time on the number of vehicles used under different values of uncertainty budget.

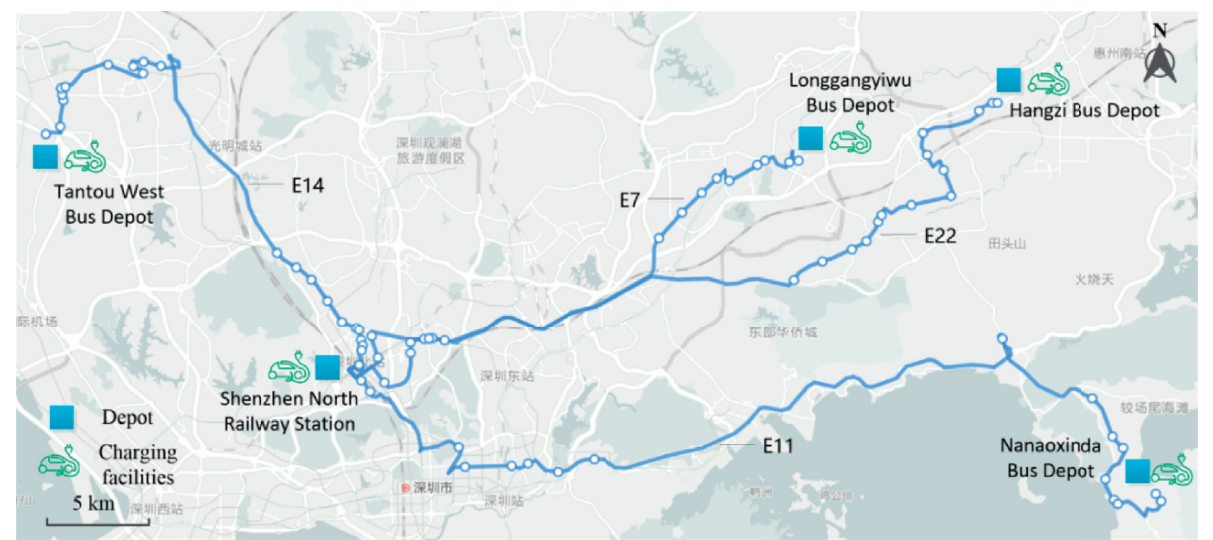

(a)

Figure 8: Continued. 


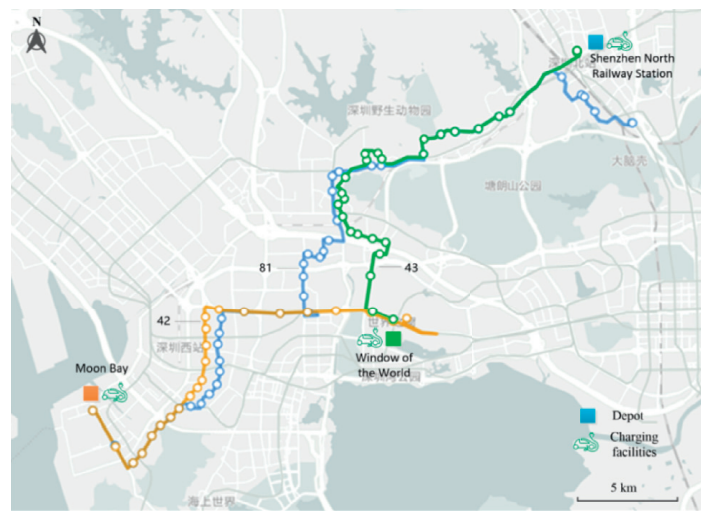

(b)

Figure 8: A display of the route layout of (a) case I and (b) case II.

TABLE 4: Computational results of the BP on multiroute instances.

\begin{tabular}{|c|c|c|c|c|c|c|}
\hline Case & $\Gamma^{t}$ & Obj. & $\# \mathrm{~V}$ & Charging amount (kWh) & Run time (s) & BP gap (\%) \\
\hline \multirow[t]{4}{*}{ Case I } & 0 & 81638.3 & 73 & 11141.3 & 392 & 1.02 \\
\hline & 1 & 92313.4 & 76 & 16313.4 & 398 & 0.96 \\
\hline & 2 & 114236.0 & 76 & 17522.4 & 676 & 1.10 \\
\hline & 3 & 132200.5 & 76 & 18247.8 & 590 & 1.32 \\
\hline \multirow[t]{4}{*}{ Case II } & 0 & 69279.0 & 64 & 6895.6 & 6248 & 1.89 \\
\hline & 1 & 76556.2 & 64 & 11178.4 & $\begin{array}{l}8979 \\
8261\end{array}$ & 1.84 \\
\hline & 2 & 86540.8 & 64 & 13533.2 & 8261 & 0.12 \\
\hline & 3 & 98607.3 & 64 & 13444.1 & 6350 & 1.06 \\
\hline
\end{tabular}

TABLE 5: Robustness of the single-route schedules.

\begin{tabular}{|c|c|c|c|c|}
\hline \multirow{2}{*}{ Number of trips } & \multirow{2}{*}{$\Gamma^{t}$} & \multicolumn{2}{|c|}{ Infeasibility rate (\%) } & \multirow{2}{*}{ PoR (\%) } \\
\hline & & Trip chain & Schedule & \\
\hline \multirow[t]{4}{*}{96} & 0 & 0.94 & 19.27 & - \\
\hline & 1 & 0.00 & 0.00 & 9.52 \\
\hline & 2 & 0.00 & 0.00 & 15.41 \\
\hline & 3 & 0.00 & 0.00 & 30.77 \\
\hline \multirow[t]{4}{*}{112} & 0 & 7.20 & 87.31 & - \\
\hline & 1 & 0.00 & 0.05 & 3.76 \\
\hline & 2 & 0.00 & 0.00 & 14.87 \\
\hline & 3 & 0.00 & 0.00 & 24.06 \\
\hline \multirow[t]{4}{*}{154} & 0 & 5.65 & 92.08 & - \\
\hline & 1 & 0.01 & 0.26 & 1.25 \\
\hline & 2 & 0.02 & 0.70 & 13.28 \\
\hline & 3 & 0.03 & 1.04 & 19.79 \\
\hline \multirow[t]{4}{*}{276} & 0 & 9.81 & 99.86 & - \\
\hline & 1 & 0.00 & 0.00 & 16.14 \\
\hline & 2 & 0.00 & 0.00 & 31.49 \\
\hline & 3 & 0.00 & 0.00 & 43.06 \\
\hline
\end{tabular}

range. Figure 9 shows the changes of the objective and penalty per 100 trip chains under different values of $\Gamma^{t}$. With the increase of $\Gamma^{t}$, the objective increase while the penalty decreases. This result coincides with that in Table 3, indicating that the schedules with $\Gamma^{t}=0$ and $\Gamma^{t}=1$ are Pareto optimal solutions.

Table 6 presents the robustness evaluation results of the multiroute scheduling cases. When $\Gamma^{t}=0$, about $4.09 \%$ and 

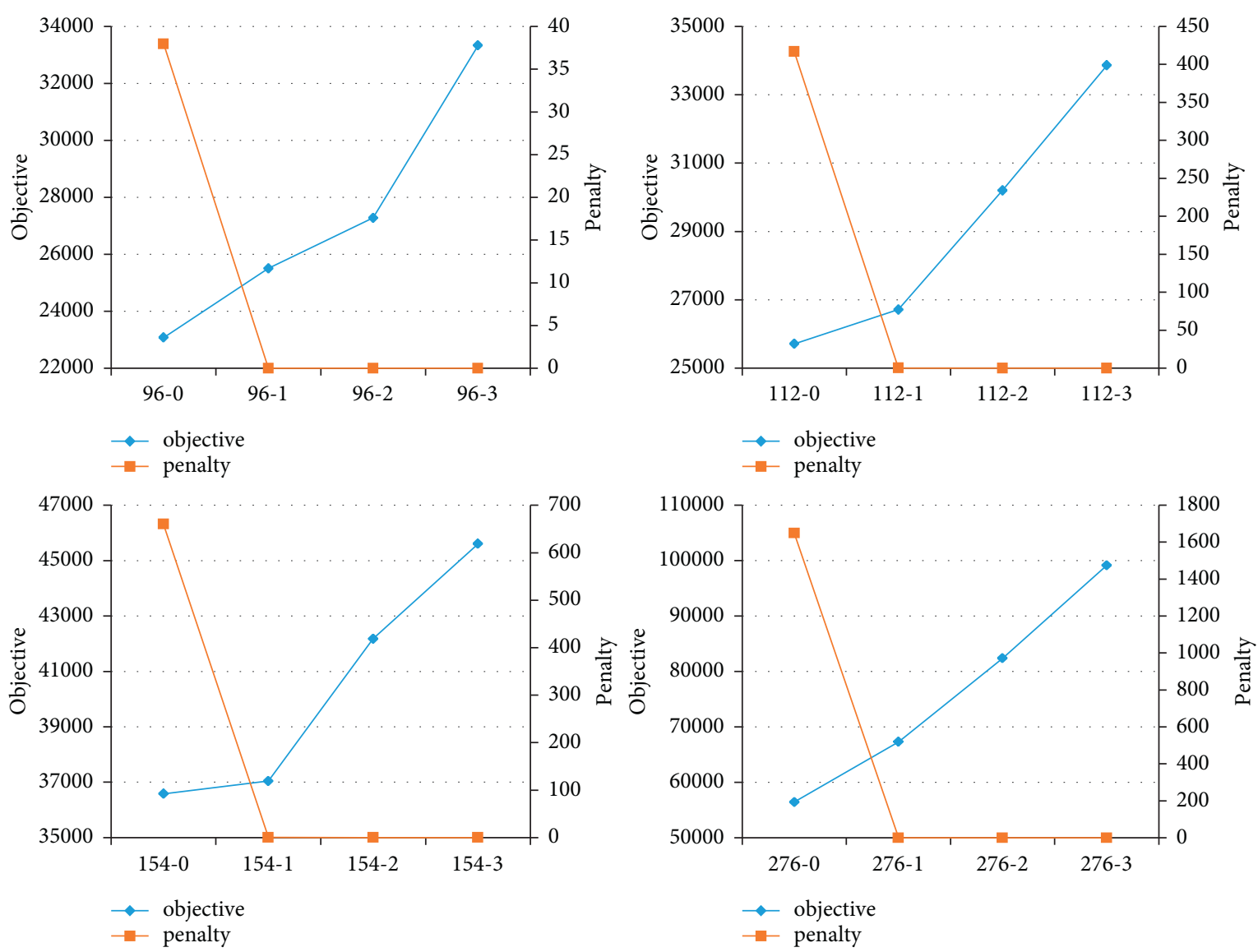

Figure 9: Objective and penalty under different values of $\Gamma^{t}$ in single-route cases.

TABLE 6: Robustness of the multiroute schedules.

\begin{tabular}{|c|c|c|c|c|}
\hline \multirow{2}{*}{ Case } & \multirow{2}{*}{$\Gamma^{t}$} & \multicolumn{2}{|c|}{ Infeasibility rate $(\%)$} & \multirow{2}{*}{ PoR $(\%$} \\
\hline & & Trip chain & Schedule & \\
\hline \multirow[t]{4}{*}{ Case I } & 0 & 4.09 & 98.84 & - \\
\hline & 1 & 0.00 & 0.00 & 10.49 \\
\hline & 2 & 0.00 & 0.00 & 19.19 \\
\hline & 3 & 0.00 & 0.00 & 13.59 \\
\hline \multirow[t]{4}{*}{ Case II } & 0 & 3.12 & 95.36 & - \\
\hline & 1 & 0.00 & 0.00 & 8.48 \\
\hline & 2 & 0.00 & 0.00 & 11.54 \\
\hline & 3 & 0.00 & 0.00 & 12.24 \\
\hline
\end{tabular}

$3.12 \%$ of the simulated trip chains experience delay in cases I and II, respectively. When $\Gamma^{t}=1$, the infeasibility rate drops to zero while the objective increases by $10.49 \%$ and $8.48 \%$ in cases I and II, respectively. We also calculated the penalty of the trips chains incurred by the violation of the trip start time window and battery SoC safety range. Figure 10 shows the changes of the objective and penalty per 100 trip chains under different values of $\Gamma^{t}$. With the increase of $\Gamma^{t}$, the objective increases while the penalty decreases. The results indicate that the schedules with $\Gamma^{t}=0$ and $\Gamma^{t}=1$ are Pareto optimal solutions.
Based on the above evaluation results, we can make the following conclusions:

(i) The infeasibility rate of the deterministic solution is significantly higher than that of the robust solutions in the simulation.

(ii) The schedule robustness can be greatly improved at the expense of an increase in the total operational cost.

(iii) Trade-off between the infeasibility rate and PoR is needed to choose a proper value of uncertainty budget when generating robust schedules. 


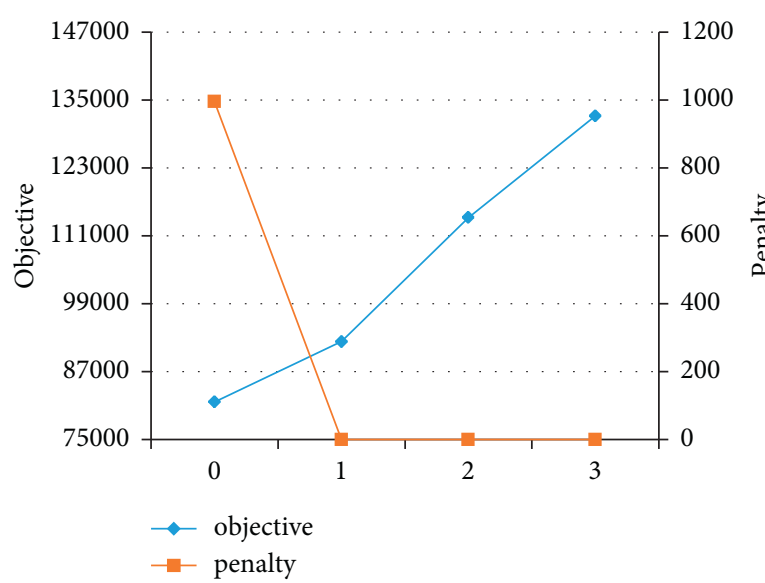

(a)

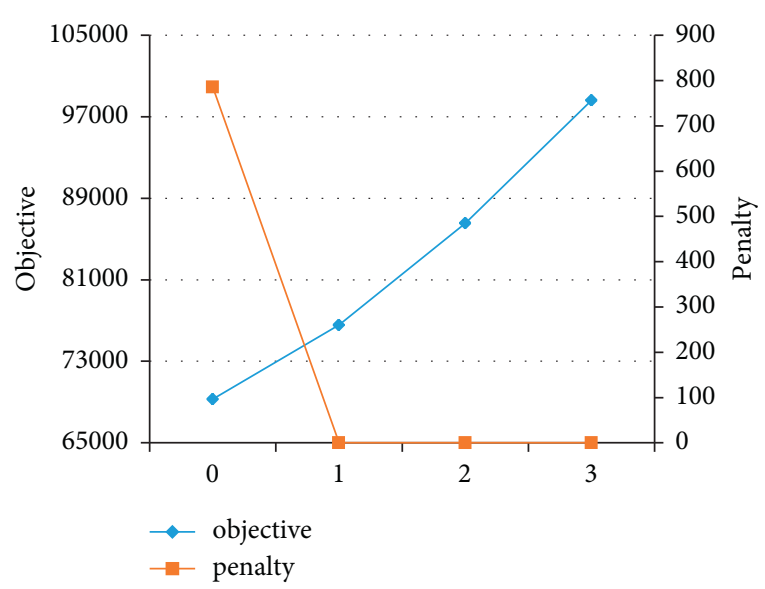

(b)

Figure 10: Objective and penalty under different values of $\Gamma^{t}$ in multiroute cases. (a) Case I. (b) Case II.

\section{Conclusion}

In this paper, an R-MD-EVSP model and BP algorithm are introduced as a novel approach for e-bus scheduling under travel time uncertainty. Partial charging and trip start time window are taken into consideration. The cardinality constrained uncertainty set was adopted to control the level of uncertainty. The proposed methodology was tested on the real-world transit operation cases in Shenzhen including single-route and multiroute cases. The size of the instances varies from 62 to 466 timetabled trips. The results showed that the $\mathrm{BP}$ algorithm is able to generate provably highquality solutions efficiently for large-scale instances. With the increase of the uncertainty budget, the planned charging amount increases to protect the schedule against the variation of energy consumption when the number of e-buses used stays the same; the charging amount can be reduced when the number of vehicles used increases. Additionally, a sensitivity analysis of the trip start buffer time was carried out. The analysis showed that allowing for trip start buffer time can increase time flexibility of the schedule and save the operational cost in some scenarios. The robustness of the schedules was evaluated through Monte Carlo simulation based on the randomly generated trip time data. The results showed that the infeasibility rate of the deterministic solution is significantly higher than that of the robust solutions. Applying the robust schedules can effectively protect against trip time and energy consumption variation at the expense of an increase in the operational cost. Therefore, transit operators need to trade off between the infeasibility rate and PoR to choose a proper value of uncertainty budget when adopting robust schedules.

In future research studies, more realistic battery charging and energy consumption process should to be considered in the robust e-bus scheduling model. Besides, heuristic methods are needed to solve the problem instances of larger scale. Furthermore, different kinds of uncertainty sets can be considered to control the trip time variation. Trip time and energy consumption can be described by independent uncertainty sets, accounting for more general e-bus operation cases.

\section{Data Availability}

The data used to support the findings of this study are available from the corresponding author upon request.

\section{Conflicts of Interest}

The authors declare that they have no conflicts of interest.

\section{Acknowledgments}

This research was supported in part by the Basic Research Program of Shenzhen Science and Technology Innovation Committee (JCYJ20180307123910003), Scientific Research Start-Up Funds of Tsinghua Shenzhen International Graduate School (QD2021007N), and National Natural Science Foundation of China (61673233).

\section{References}

[1] J.-Q. Li, "Battery-electric transit bus developments and operations: a review," International Journal of Sustainable Transportation, vol. 10, no. 3, pp. 157-169, 2016.

[2] Y. Shen, J. Xu, and J. Li, "A probabilistic model for vehicle scheduling based on stochastic trip times," Transportation Research Part B: Methodological, vol. 85, pp. 19-31, 2016.

[3] D. Huisman, R. Freling, and A. P. M. Wagelmans, "A robust solution approach to the dynamic vehicle scheduling problem," Transportation Science, vol. 38, no. 4, pp. 447-458, 2004.

[4] M. Naumann, L. Suhl, and S. Kramkowski, "A stochastic programming approach for robust vehicle scheduling in public bus transport," Procedia - Social and Behavioral Sciences, vol. 20, pp. 826-835, 2011.

[5] F. He, J. Yang, and M. Li, "Vehicle scheduling under stochastic trip times: an approximate dynamic programming approach," Transportation Research Part C: Emerging Technologies, vol. 96, pp. 144-159, 2018.

[6] X. Tang, X. Lin, and F. He, "Robust scheduling strategies of electric buses under stochastic traffic conditions," Transportation Research Part C: Emerging Technologies, vol. 105, pp. 163-182, 2019. 
[7] Y. Bie, J. Ji, X. Wang, and X. Qu, "Optimization of Electric Bus Scheduling Considering Stochastic Volatilities in Trip Travel Time and Energy Consumption," Computer-Aided Civil and Infrastructure Engineering, vol. 36, no. 12, pp. 1530-1548, 2021.

[8] A. Bertossi, P. Carraresi, and G. Gallo, "On some matching problems arising in vehicle scheduling models," Networks, vol. 17, no. 3, pp. 271-281, 1989.

[9] J.-Q. Li, "Transit bus scheduling with limited energy," Transportation Science, vol. 48, no. 4, pp. 521-539, 2014.

[10] Y. Yang, M. Guan, and J. Ma, "Battery electric transit bus scheduling problem based on column generation approach," Journal of Transportation Systems Engineering and Information Technology, vol. 16, pp. 198-204, 2016.

[11] T. Liu and A. Ceder, "Battery-electric transit vehicle scheduling with optimal number of stationary chargers," Transportation Research Part C: Emerging Technologies, vol. 114, pp. 118-139, 2020.

[12] L. Li, H. K. Lo, and F. Xiao, "Mixed bus fleet scheduling under range and refueling constraints," Transportation Research Part C: Emerging Technologies, vol. 104, pp. 443-462, 2019.

[13] M. Rinaldi, E. Picarelli, A. D’Ariano, and F. Viti, "Mixed-fleet single-terminal bus scheduling problem: modelling, solution scheme and potential applications," Omega, vol. 96, Article ID 102070, 2020.

[14] E. Yao, T. Liu, T. Lu, and Y. Yang, "Optimization of electric vehicle scheduling with multiple vehicle types in public transport," Sustainable Cities and Society, vol. 52, Article ID 101862, 2020.

[15] L. Zhang, S. Wang, and X. Qu, "Optimal electric bus fleet scheduling considering battery degradation and non-linear charging profile," Transportation Research Part E: Logistics and Transportation Review, vol. 154, Article ID 102445, 2021.

[16] A. Zhang, T. Li, R. Tu et al., "The effect of nonlinear charging function and line change constraints on electric bus scheduling," Promet - Traffic \& Transportation, vol. 33, no. 4, pp. 527-538, 2021.

[17] M. Wen, E. Linde, S. Ropke, P. Mirchandani, and A. Larsen, "An adaptive large neighborhood search heuristic for the Electric Vehicle Scheduling Problem," Computers \& Operations Research, vol. 76, pp. 73-83, 2016.

[18] M. E. van Kooten Niekerk, J. M. van den Akker, and J. A. Hoogeveen, "Scheduling electric vehicles," Public Transport, vol. 9, no. 1-2, pp. 155-176, 2017.

[19] M. Koháni and M. Kohánia, "Exact approach to the electric bus fleet scheduling," Transportation Research Procedia, vol. 40, pp. 1380-1387, 2019.

[20] X. Li, T. Wang, L. Li et al., "Joint optimization of regular charging electric bus transit network schedule and stationary charger deployment considering partial charging policy and time-of-use electricity prices," Journal of Advanced Transportation, vol. 2020, pp. 1-16, 2020.

[21] Ş. Yıldırım and B. Yıldız, "Electric bus fleet composition and scheduling," Transportation Research Part C: Emerging Technologies, vol. 129, Article ID 103197, 2021.

[22] N. Qin, A. Gusrialdi, R. Paul Brooker, and A. T. Raissi, "Numerical analysis of electric bus fast charging strategies for demand charge reduction," Transportation Research Part A: Policy and Practice, vol. 94, pp. 386-396, 2016.

[23] Y. Wang, Y. Huang, J. Xu, and N. Barclay, "Optimal recharging scheduling for urban electric buses: a case study in Davis," Transportation Research Part E: Logistics and Transportation Review, vol. 100, pp. 115-132, 2017.
[24] Y. He, Z. Liu, and Z. Song, "Optimal charging scheduling and management for a fast-charging battery electric bus system," Transportation Research Part E: Logistics and Transportation Review, vol. 142, Article ID 102056, 2020.

[25] K. Liu, H. Gao, Z. Liang, M. Zhao, and C. Li, "Optimal charging strategy for large-scale electric buses considering resource constraints," Transportation Research Part D: Transport and Environment, vol. 99, Article ID 103009, 2021.

[26] M. Salicrú, C. Fleurent, and J. M. Armengol, "Timetable-based operation in urban transport: run-time optimisation and improvements in the operating process," Transportation Research Part A: Policy and Practice, vol. 45, pp. 721-740, 2011.

[27] J. Xu and Y. Shen, "Setting scheduled trip time based on AVL data," Journal of Transportation Systems Engineering and Information Technology, vol. 12, no. 5, pp. 40-45, 2012.

[28] D. Bertsimas and M. Sim, "Robust discrete optimization and network flows," Mathematical Programming, vol. 98, no. 1, pp. 49-71, 2003.

[29] A. Agra, M. Christiansen, R. Figueiredo, L. M. Hvattum, M. Poss, and C. Requejo, "The robust vehicle routing problem with time windows," Computers \& Operations Research, vol. 40, no. 3, pp. 856-866, 2013.

[30] P. Munari, A. Moreno, J. De La Vega, D. Alem, J. Gondzio, and R. Morabito, "The robust vehicle routing problem with time windows: compact formulation and branch-price-andcut method," Transportation Science, vol. 53, no. 4, pp. 1043-1066, 2019.

[31] D. Lu and F. Gzara, "The robust vehicle routing problem with time windows: solution by branch and price and cut," European Journal of Operational Research, vol. 275, no. 3, pp. 925-938, 2019.

[32] S. Braaten, O. Gjønnes, L. M. Hvattum, and G. Tirado, "Heuristics for the robust vehicle routing problem with time windows," Expert Systems with Applications, vol. 77, pp. 136-147, 2017.

[33] C. Hu, J. Lu, X. Liu, and G. Zhang, "Robust vehicle routing problem with hard time windows under demand and travel time uncertainty," Computers \& Operations Research, vol. 94, pp. 139-153, 2018.

[34] S. Pelletier, O. Jabali, and G. Laporte, "The electric vehicle routing problem with energy consumption uncertainty," Transportation Research Part B: Methodological, vol. 126, pp. 225-255, 2019.

[35] S. Irnich and G. Desaulniers, "Shortest path problems with resource constraints," in Column Generation, G. Desaulniers, J. Desrosiers, and M. M. Solomon, Eds., Springer, Boston, MA, USA, pp. 33-65, 2005. 